\title{
Factorial Grothendieck Polynomials
}

\author{
Peter J. McNamara \\ Department of Mathematics and Statistics \\ University of Sydney, NSW 2006, Australia \\ petermcn@maths. usyd.edu.au
}

Submitted: Aug 10, 2005; Accepted: Jan 8, 2006; Published: Aug 10, 2006

Mathematics Subject Classifications: 05E05

\begin{abstract}
In this paper, we study Grothendieck polynomials indexed by Grassmannian permutations from a combinatorial viewpoint. We introduce the factorial Grothendieck polynomials which are analogues of the factorial Schur functions, study their properties, and use them to produce a generalisation of a Littlewood-Richardson rule for Grothendieck polynomials.
\end{abstract}

\section{Introduction}

Let $x=\left(x_{1}, \ldots, x_{n}\right)$ be a set of variables, $\beta$ a parameter and $\theta$ a skew Young diagram whose columns have at most $n$ boxes. A set-valued $\theta$-tableau $T$ is obtained by placing subsets of $[n]=\{1, \ldots, n\}$ (a notation used throughout) into the boxes of $T$ in such a way that the rows weakly increase while the columns strictly increase. More precisely, in each cell $\alpha$ of $\theta$, place a non-empty set $T(\alpha) \subset[n]$ so that if $\alpha$ is immediately to the left of $\beta$ then $\max (T(\alpha)) \leq \min (T(\beta))$, while if $\alpha$ is immediately above $\beta$, then $\max (T(\alpha))<\min (T(\beta))$. An example of such a $(4,4,2,1) /(1)$-tableau is given by the following:

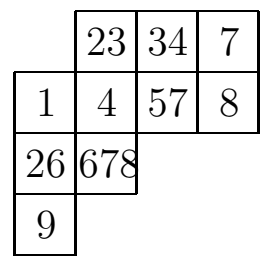

Given a skew diagram $\theta$, the (ordinary) Grothendieck polynomial $G_{\theta}(x)$ is defined by

$$
G_{\theta}(x)=\sum_{T} \beta^{|T|-|\theta|} \prod_{\substack{\alpha \in \theta \\ r \in T(\alpha)}} x_{r}
$$

where the sum is over all set-valued $\theta$-tableaux $T$. 
In a different form, the Grothendieck polynomials were first introduced by Lascoux and Schützenberger [10] as representatives for $K$-theory classes determined by structure sheaves of Schubert varieties. Since then, their properties were studied by Fomin and Kirillov [4, 5], Lenart [11], Buch [2]. In particular, the latter paper contains the above combinatorial description of Grothendieck polynomials in terms of tableaux, similar to that for the Schur polynomials. It is this formulation which we use as the basis for our approach to the study of Grothendieck polynomials in this paper.

The major focus of this paper is the introduction and study of what we shall call the factorial Grothendieck polynomials. They generalise (1.1) by introducing a second set of variables $\left(a_{i}\right)_{i \in \mathbb{Z}}$ and we define the factorial Grothendieck polynomial in $n$ variables $\left(x_{1}, x_{2}, \ldots, x_{n}\right)$ by

$$
G_{\theta}(x \mid a)=\sum_{T} \beta^{|T|-|\theta|} \prod_{\substack{\alpha \in \theta \\ r \in T(\alpha)}} x_{r} \oplus a_{r+c(\alpha)}
$$

where $c(\alpha)$ is the content of the cell $\alpha$, defined by $c(i, j)=j-i$, and again the sum is over all set-valued $\theta$-tableaux $T$.

These factorial Grothendieck polynomials specialise in two different ways, firstly by setting $a_{i}=0$ for all $i$ to obtain the ordinary Grothendieck polynomials, and secondly by setting $\beta=0$ to obtain the factorial Schur polynomials as studied in [14]. Of these two families of polynomials obtained via specialisation, the theory and properties of the factorial Grothendieck polynomials appear to mimic more closely that of the factorial Schur polynomials.

It can be shown and indeed is shown in this paper (Theorem 4.6) that the factorial Grothendieck polynomials $G_{\lambda}(x \mid a)$ with $\lambda$ running over the (non-skew) partitions with length at most $n$ form a basis of the ring of symmetric polynomials in $x_{1}, \ldots, x_{n}$. Hence, we can define the coefficients $c_{\theta \mu}^{\nu}(\beta, a)$ by the expansion

$$
G_{\theta}(x \mid a) G_{\mu}(x \mid a)=\sum_{\nu} c_{\theta \mu}^{\nu}(\beta, a) G_{\nu}(x \mid a)
$$

In order to obtain a rule describing these coefficients, we closely follow the method of Molev and Sagan [14], ${ }^{1}$ exploiting the similarities between the factorial Grothendieck polynomials and factorial Schur polynomials. This approach relies on properties peculiar to the factorial versions of the polynomials which enable a recurrence relation for the coefficients to be determined, though there are also some characteristics unique to the Grothendieck case, most notably in section 4.2 .

We present three solutions to the recurrence relation obtained for the coefficients. The first of these is a general formula where $G_{\theta}(x \mid a)$ in (1.2) is replaced by an arbitrary symmetric polynomial. The second is a full solution in the case where $\theta$ has no two boxes in the same column, which is essentially a Pieri rule for factorial Grothendieck polynomials. The third solution is a partial rule for arbitrary $\theta$ obtained by specialising certain variables to zero.

\footnotetext{
${ }^{1}$ We are grateful to Anatol Kirillov for suggesting to apply this method to the Grothendieck polynomials.
} 
Out of the third solution, an application of the theory of factorial Grothendieck polynomials to that of ordinary Grothendieck polynomials is obtained. This consists of a combinatorial rule for the calculation of the coefficients $c_{\theta \mu}^{\nu}(\beta, 0)$, generalising a previous result of Buch [2]. In order to formulate the rule, define the column word of a set-valued tableau $T$ as the sequence obtained by reading the entries of $T$ from top to bottom in successive columns starting from the right most column with the rule that the entries of a particular box are read in the decreasing order. As an example, the column word of the tableau depicted earlier in the introduction is 7843753248761629 .

We write $\lambda \rightarrow \mu$ if $\mu$ is obtained by adding one box to $\lambda$. If $r$ is the row number of the box added to $\lambda$ to create $\mu$ then write $\lambda \stackrel{r}{\rightarrow} \mu$. A set-valued tableau $T$ fits a sequence $R(\mu, \nu)$ of partitions

$$
\mu=\rho^{(0)} \stackrel{r_{1}}{\longrightarrow} \rho^{(1)} \stackrel{r_{2}}{\longrightarrow} \cdots \stackrel{r_{l}}{\longrightarrow} \rho^{(l)}=\nu
$$

if the column word of $T$ coincides with $r_{1} \ldots r_{l}$. With this notation, we have

Theorem The coefficient $c_{\theta \mu}^{\nu}(\beta, 0)$ is equal to $\beta^{|\nu|-|\mu|-|\theta|}$ times the number of set-valued $\theta$-tableaux $T$ such that $T$ fits a sequence $R(\mu, \nu)$.

In the particular cases where $\theta=\lambda$ is normal, or $\mu=\emptyset$, our rule coincides with the one previously given by Buch [2]. Note also that if $\beta$ is specialised to 0 then $G_{\theta}(x)$ becomes the Schur polynomial $s_{\theta}(x)$ so that the values $c_{\theta \mu}^{\nu}(0,0)$ coincide with the LittlewoodRichardson coefficients $c_{\theta \mu}^{\nu}$ defined by the expansion

$$
s_{\theta}(x) s_{\mu}(x)=\sum_{\nu} c_{\theta \mu}^{\nu} s_{\nu}(x)
$$

The coefficients $c_{\lambda \mu}^{\nu}$ with a non-skew partition $\lambda$ can be calculated by the classical Littlewood-Richardson rule [8] and its various versions; see e.g. Macdonald [13], Sagan [17]. In the case where $\theta$ is skew, a rule for calculation of $c_{\theta \mu}^{\nu}$ is given by James and Peel [7] and Zelevinsky [18] in terms of combinatorial objects called pictures. There is also a short proof of a generalised Littlewood-Richardson rule for Schur polynomials provided by Gasharov [6], which raises the question as to whether an analogue exists for Grothendieck polynomials. A different derivation of such a rule is given by Molev and Sagan [14], where a factorial analogue of the Schur functions was used.

The results given by Buch in [2] are shown to be an immediate consequence of this new rule. As for the question of providing a complete description of the Littlewood-Richardson rule for factorial Grothendieck polynomials, this remains unanswered.

In the last two sections, we turn away from the combinatorial approach to Grothendieck polynomials used elsewhere in this paper and consider the so-called double Grothendieck polynomials defined via isobaric divided difference operators. These chapters work towards, and eventually prove, the existence of a relationship between these previously studied double Grothendieck polynomials and the factorial Grothendieck polynomials introduced here. 


\section{Preliminaries}

\subsection{Partitions}

A partition $\lambda=\left(\lambda_{1}, \lambda_{2}, \ldots, \lambda_{l}\right)$ is a finite non-increasing sequence of positive integers, $\lambda_{1} \geq \lambda_{2} \geq \cdots \geq \lambda_{l}>0$. The number of parts $l$, is called the length of $\lambda$, and denoted $\ell(\lambda)$. Throughout this paper, we shall frequently be dealing with the set of partitions $\lambda$ for which $\ell(\lambda) \leq n$ for some fixed positive integer $n$. Then, if $\ell(\lambda)<n$ we shall append zeros to the end of $\lambda$ by defining $\lambda_{k}=0$ if $\ell(\lambda)<k \leq n$ so we can treat $\lambda$ as a sequence $\left(\lambda_{1}, \lambda_{2}, \ldots, \lambda_{n}\right)$ of $n$ non-negative integers.

Denote by $|\lambda|$ the weight of the partition $\lambda$, defined as the sum of its parts, $|\lambda|=$ $\sum_{i=1}^{\ell(\lambda)} \lambda_{i}$

An alternative notation for a partition is to write $\lambda=\left(1^{m_{1}} 2^{m_{2}} \ldots\right)$ where $m_{i}$ is the number of indices $j$ for which $\lambda_{j}=i$. In such notation, if $m_{i}=0$ for some $i$, then we omit it from our notation. So for example we can succinctly write the partition consisting of $n$ parts each equal to $k$ as $\left(k^{n}\right)$.

The Young diagram of a partition $\lambda$ is formed by left-aligning $\ell(\lambda)$ rows of boxes, or cells, where the $i$-th row (counting from the top) contains $\lambda_{i}$ boxes.

We identify a partition with its Young diagram.

Say $\lambda \rightarrow \mu$ if $\mu$ is obtained by adding one box to $\lambda$. If $r$ is the row number of the box added to $\lambda$ to create $\mu$ then write $\lambda \stackrel{r}{\rightarrow} \mu$.

By reflecting the diagram of $\lambda$ in the main diagonal, we get the diagram of another partition, called the conjugate partition, and denoted $\lambda^{\prime}$. Alternatively and equivalently, we can define $\lambda^{\prime}$ by $\lambda_{j}^{\prime}=\#\left\{i \mid \lambda_{i} \geq j\right\}$.

The main ordering of partitions which we make use of is that of containment ordering. We say $\lambda \subset \mu$ if the Young diagram of $\lambda$ is a subset of the Young diagram of $\mu$.

The other ordering which we make mention of is dominance ordering. We say $\lambda \triangleright \mu$ if $\lambda_{1}+\cdots+\lambda_{k} \geq \mu_{1}+\cdots+\mu_{k}$ for all $k$.

Suppose we have two partitions $\lambda, \mu$ with $\lambda \supset \mu$. Then we may take the set-theoretic difference of their Young diagrams and define the skew partition $\theta=\lambda / \mu$ to be this diagram. Note that every partition is also a skew partition since $\lambda=\lambda / \phi$ where $\phi$ is the empty partition.

The weight of $\theta$ is the number of boxes it contains: $|\theta|=|\lambda / \mu|=|\lambda|-|\mu|$.

With regard to notation, the use of $\theta$ shall signify that we are dealing with a skew partition, while other Greek letters employed shall refer exclusively to partitions.

\subsection{Tableaux}

Let $\theta$ be a skew partition. We introduce a co-ordinate system of labelling cells of $\theta$ by letting $(i, j)$ be the intersection of the $i$-th row and the $j$-th column. Define the content of the cell $\alpha=(i, j)$ to be $c(\alpha)=j-i$.

In each cell $\alpha$ of $\theta$, place a non-empty set $T(\alpha) \subset[n]=\{1,2, \ldots, n\}$ (a notation we shall use throughout), such that entries are non-decreasing along rows and strictly increas- 
ing down columns. In other words, if $\alpha$ is immediately to the left of $\beta$ then $\max (T(\alpha)) \leq$ $\min (T(\beta))$, while if $\alpha$ is immediately above $\beta$, then $\max (T(\alpha))<\min (T(\beta))$.

An example of such a $(4,4,2,1) /(1)$-tableau is given in the Introduction.

Such a combinatorial object $T$ is called a semistandard set-valued $\theta$-tableau. If the meaning is obvious from the context, we shall often drop the adjectives semistandard and set-valued. $\theta$ is said to be the shape of $T$, which we denote by $\operatorname{sh}(T)$.

Define an entry of $T$ to be a pair $(r, \alpha)$ where $\alpha \in \theta$ is a cell and $r \in T(\alpha)$. Let $|T|$ denote the number of entries in $T$.

Define an ordering $\prec$ on the entries of $T$ by $(r,(i, j)) \prec\left(r^{\prime},\left(i^{\prime}, j^{\prime}\right)\right)$ if $j>j^{\prime}$, or $j=j^{\prime}$ and $i<i^{\prime}$, or $(i, j)=\left(i^{\prime}, j^{\prime}\right)$ and $r>r^{\prime}$. On occasion, we shall abbreviate this to $r \prec r^{\prime}$.

So any two entries of $T$ are comparable under this order, and if we write all the entries of $T$ in a chain $\left(r_{1}, \alpha_{1}\right) \prec\left(r_{2}, \alpha_{2}\right) \prec \ldots \prec\left(r_{|T|}, \alpha_{|T|}\right)$, then this is equivalent to reading them one column at a time from right to left, from top to bottom within each column, and from largest to smallest in each cell. Writing the entries in this way, we create a word $r_{1} r_{2} \ldots r_{|T|}$, called the column word of $T$, and denoted $c(T)$.

\subsection{Symmetric functions}

Here we define the monomial symmetric function $m_{\lambda}$ and the elementary symmetric function $e_{k}$ in $n$ variables $\left(x_{1}, x_{2}, \ldots, x_{n}\right)$.

For a partition $\lambda=\left(\lambda_{1}, \lambda_{2}, \ldots, \lambda_{n}\right)$, define the monomial symmetric function $m_{\lambda}$ by

$$
m_{\lambda}(x)=\sum \prod_{i=1}^{n} x_{\pi(i)}^{\lambda_{i}}
$$

where the sum runs over all distinct values of $\prod_{i=1}^{n} x_{\pi(i)}^{\lambda_{i}}$ that are attainable as $\pi$ runs over the symmetric group $S_{n}$.

As an example, if $n=3$, then we have $m_{(22)}\left(x_{1}, x_{2}, x_{3}\right)=x_{1}^{2} x_{2}^{2}+x_{2}^{2} x_{3}^{2}+x_{3}^{2} x_{1}^{2}$.

The elementary symmetric function $e_{k}$ can now be defined as $e_{k}=m_{\left(1^{k}\right)}$.

The monomial symmetric functions $m_{\lambda}$, where $\lambda$ runs over all partitions with $\ell(\lambda) \leq n$, form a basis for the ring of symmetric polynomials in $n$ variables, $\Lambda_{n}$.

We will stick with convention and use $\Lambda_{n}$ to denote the ring of symmetric polynomials in $n$ variables over $\mathbb{Z}$. However, we will often wish to change the ring of coefficients, so will often work in $\Lambda_{n} \otimes_{\mathbb{Z}} R$ for some ring $R$. As we shall only ever consider tensor products over $\mathbb{Z}$, the subscript $\mathbb{Z}$ is to be assumed whenever omitted.

\section{Ordinary Grothendieck Polynomials}

Before starting our work on the factorial Grothendieck polynomials, first we present some of the theory of the ordinary Grothendieck polynomials. 
Definition 3.1. Given a skew diagram $\theta$, a field $\mathbb{F}, \beta$ an indeterminate over $\mathbb{F}$, we define the ordinary Grothendieck polynomial $G_{\theta}(x) \in \mathbb{F}(\beta)\left[x_{1}, \ldots, x_{n}\right]$ by

$$
G_{\theta}(x)=\sum_{T} \beta^{|T|-|\theta|} \prod_{\substack{\alpha \in \theta \\ r \in T(\alpha)}} x_{r}
$$

where the sum is over all semistandard set-valued $\theta$-tableau $T$.

Remark 3.2. In the existing literature, Grothendieck polynomials are often only presented in the case $\beta=-1$ as a consequence of their original geometric meaning. The case of arbitrary $\beta$ has been previously studied in [4] and [5], though there is essentially little difference between the two cases, as can be seen by replacing $x_{i}$ with $-x_{i} / \beta$ in (3.1) for all $i$.

Example 3.3. Calculation of $G_{(1)}(x)$.

We can have any nonempty subset of $[n]$ in the single available cell of $T$, so we have

$$
G_{(1)}(x)=\sum_{\substack{S \subset[n] \\ S \neq \phi}} \beta^{|S|-1} \prod_{i \in S} x_{i}=\sum_{j=1}^{n} \beta^{j-1} \sum_{\substack{S \subset[n] \\|S|=j}} \prod_{i \in S} x_{i}=\sum_{j=1}^{n} \beta^{j-1} e_{j}(x) .
$$

where the $e_{j}$ are the elementary symmetric functions. Hence,

$$
1+\beta G_{(1)}(x)=\sum_{j=0}^{n} \beta^{j} e_{j}(x)=\prod_{i=1}^{n}\left(1+\beta x_{i}\right)=\Pi(x) .
$$

where for any sequence $y=\left(y_{1}, y_{2}, \ldots, y_{n}\right)$, we denote the product $\prod_{i=1}^{n}\left(1+\beta y_{i}\right)$ by $\Pi(y)$.

At this stage we will merely state, rather than prove the following important theorem about ordinary Grothendieck polynomials, as it is proven in greater generality in Theorems 4.3 and 4.9 of the following section.

Theorem 3.4. The ordinary Grothendieck polynomial $G_{\theta}(x)$ is symmetric in $x_{1}, \ldots, x_{n}$, and furthermore the polynomials $\left\{G_{\lambda}(x) \mid \ell(\lambda) \leq n\right\}$ comprise a basis for the ring of symmetric polynomials in $n$ variables $\Lambda_{n} \otimes \mathbb{F}(\beta)$.

For a skew-partition $\theta$, and partitions $\mu, \nu$ with $\ell(\nu) \leq n$, we define the coefficients $c_{\theta \mu}^{\nu} \in \mathbb{F}(\beta)$ by

$$
G_{\theta}(x) G_{\mu}(x)=\sum_{\nu} c_{\theta \mu}^{\nu} G_{\nu}(x) .
$$

The above theorem shows that these coefficients are well defined.

Before moving onto an important result from the theory of ordinary Grothendieck polynomials, we present two insertion algorithms which play an integral role in the proof. Buch [2] presents a similar column-based insertion algorithm.

First we present a forward row insertion algorithm. As input, this algorithm takes a set $S \subset[n]$ and a semistandard, set-valued row $R$ and produces as output a row $R^{\prime}$ and a set $S^{\prime}$. 
Algorithm 3.5 (Forward row insertion algorithm). For all $s \in S$, we perform the following operations simultaneously:

Place $s$ in the leftmost cell of $R$ such that $s$ is less than all entries originally in that cell. If such a cell does not exist, then we add a new cell to the end of $R$ and place $s$ in this cell.

If there exist entries greater than $s$ occupying cells to the left of where $s$ was inserted, then remove them from $R$. Call this a type I ejection. If no such elements exist, then remove from $R$ all the original entries in the cell $s$ is inserted into and call this a type II ejection. The resulting row is $R^{\prime}$ and the set of elements removed from $R$ is $S^{\prime}$.

For example if $S=\{1,2,3,6,7,8\}$ and $R$ is the row $1,12,47,7,789,9$ then the algorithm gives:

$\begin{array}{cccccccc}124678 \rightarrow & 1 & 12 & 37 & 7 & 789 & 9 & \\ \text { Insert } & & & 12 & 46 & & 78 & \\ \text { Eject } & & 2 & 37 & & 89 & & \\ \text { Final Result } & 1 & 1 & 12 & 467 & 7 & 789 & \rightarrow 23789\end{array}$

with output $R^{\prime}=1,1,12,467,7,789$ and $S^{\prime}=\{2,3,7,8,9\}$.

We show that in this algorithm, if a number $x$ is ejected, then it is ejected from the rightmost cell in $R$ such that $x$ is strictly greater than all entries of $R^{\prime}$ in that cell.

Let $y$ be an entry of $R^{\prime}$ in the cell $x$ is ejected from, and suppose that $y \geq x$. If $y$ was, along with $x$ an original entry of $R$, then $y$ would have been ejected from $R$ at the same time that $x$ was, a contradiction. Hence $y$ was inserted from $S$ into $R$. But then, due to the criteria of which cell an entry gets inserted into, we must have $y<x$, also a contradiction. So $x$ is greater than all entries of $R^{\prime}$ in the cell it was ejected from.

Now consider a cell $\alpha \in R$ to the right of the one $x$ was ejected from, and let its maximum entry of $\alpha$ in $R^{\prime}$ be $y$. If $y$ was an original entry of $R$, then since $R$ is semistandard, $y \geq x$. Now suppose that $y$ was inserted into $R$ from $S$, and further suppose, for want of a contradiction, that $y<x$. Let $z$ be the minimal original entry in $\alpha$. Any element inserted into $\alpha$ is less than or equal to $y$, so less than $x$ and hence ejects $x$ via a type I ejection. So no type II ejections occur in $\alpha$. Now $z>y$ by our insertion rule for adding $y$, so by maximality of $y, z$ must have been ejected from $R$. Then this must have occurred via a type I ejection. To be ejected, an element $w<z$ must have been added to the right of $z$, but such a $w$ cannot be added to the right of $z$ by the conditions for insertion, a contradiction. Hence $y \geq x$.

So we have proven that if a number $x$ is ejected, then it is ejected from the rightmost cell in $R$ such that $x$ is strictly greater than all entries of $R^{\prime}$ in that cell. If an element of $S$, when inserted into $R$ does not cause any entries to be ejected, then it must have been inserted into a new cell to the right of $R^{\prime}$. We are now in a position to describe the inverse to this algorithm, which we call the reverse row insertion algorithm.

Algorithm 3.6 (Reverse Row Insertion Algorithm). The reverse insertion of a set $S^{\prime}$ into a row $R^{\prime}$, whose rightmost cell is possibly denoted special, produces as output a set $S$ and a row $R$, and is described as follows:

For all $x \in S^{\prime}$, we perform the following operations simultaneously: 
Insert $x$ in the rightmost non-special cell of $R^{\prime}$ such that $x$ is strictly greater than all entries already in that cell.

If there exist entries in $R^{\prime}$ less than $x$ in cells to the right of $x$, remove them. If this does not occur, then delete all original entries of $R^{\prime}$ in the cell in which $x$ was inserted to.

Also, remove all elements in the special cell and delete this special cell if a special cell exists.

The remaining row is $R$ and $S$ is taken to be the set of all entries removed from $R^{\prime}$.

We now present an algorithm for inserting a set $S_{0} \subset[n]$ into a semistandard set-valued tableau $T$.

Algorithm 3.7 (Forward Insertion Algorithm). Let the rows of $T$ be $R_{1}, R_{2}, \ldots$ in that order. Step $k$ of this insertion algorithm consists of inserting $S_{k-1}$ into $R_{k}$ using the forward row insertion algorithm described above, outputting the row $R_{k}^{\prime}$ and the set $S_{k}$. The resultant tableau $T^{\prime}$ with rows $R_{1}^{\prime}, R_{2}^{\prime}, \ldots$ is the output of this algorithm. Write $T^{\prime}=S \hookrightarrow T$.

Now we show that $T^{\prime}=S \hookrightarrow T$ is a semistandard set-valued tableau, and furthermore that if $T$ has shape $\lambda$ and $T^{\prime}$ has shape $\mu$, then $\lambda \rightrightarrows \mu$.

It is an immediate consequence of the nature of the row insertion algorithm that each row of $T^{\prime}$ is non-decreasing. To show that entries strictly decrease down a column, we need to look at what happens to an entry ejected from a row $R_{k}$ and inserted into $R_{k+1}$. Suppose that this entry is $a$ and is ejected from the $j$ 'th column and inserted into the $i$ 'th column of $R_{k+1}$. Then $a \in T(k, j)$ so $a<T(k+1, j)$ and hence $i \leq j$. Any entry in $T(k, i)$ greater than or equal to $a$ must also be ejected from $R_{k}$ so $T^{\prime}(k, i)<a$. Since this algorithm always decreases the entries in any given cell, the only place where semistandardness down a column needs to be checked is of the form $T^{\prime}(k, i)$ above the inserted $a$ as checked above, so $T^{\prime}$ is indeed semistandard.

In the transition from $T$ to $T^{\prime}$, clearly no two boxes can be added in the same row. Now, when a box is added, no entries are ejected from this box. We have just shown above that the path of inserted and ejected entries always moves downward and to the left, so it is impossible for entries to be added strictly below an added box, so hence no two boxes can be added in the same column, so our desired statement regarding the relative shapes of $T$ and $T^{\prime}$ is proven.

We now construct the inverse algorithm. Let $\lambda$ be a partition and suppose $T^{\prime}$ is a semistandard set-valued tableau with shape $\mu$ where $\lambda \rightrightarrows \mu$. Call a cell of $T^{\prime}$ special if it is in $\mu / \lambda$. The inverse algorithm takes as input $T^{\prime}$ as described above and produces a $\lambda$-tableau $T$ and a set $S \subset[n]$ for which $T^{\prime}=S \hookrightarrow T$.

Now, supposing we have a $\mu$-tableau $T^{\prime}$ as described above with rows $R_{1}^{\prime}, R_{2}^{\prime}, \ldots, R_{\ell(\mu)}^{\prime}$. Let $S_{\ell(\mu)}=\phi$ and form $R_{k}$ and $S_{k-1}$ by reverse inserting $S_{k}$ into $R_{k}^{\prime}$. Then $T$ is the resulting tableau consisting of rows $R_{1}, R_{2}, \ldots$ and $S=S_{0}$.

This completes our description of the necessary insertion algorithms. We note that the forward row insertion algorithm and the reverse row insertion algorithm are inverses of each other, we have constructed the inverse of the map $(S, T) \mapsto(S \hookrightarrow T)$ and hence this map is a bijection. 
The following equation is due to Lenart [11]. The proof we give however is based on the algorithm depicted above.

Say $\lambda \rightrightarrows \mu$ if $\mu / \lambda$ has all its boxes in different rows and columns (this notation also includes the case $\lambda=\mu$ ). If we want to discount the possibility that $\lambda=\mu$, then we write $\lambda \rightrightarrows^{*} \mu$.

Proposition 3.8. [11]

$$
G_{\lambda}(x) \Pi(x)=\sum_{\lambda \rightrightarrows \mu} \beta^{|\mu / \lambda|} G_{\mu}(x)
$$

Proof. We have a bijection via our insertion algorithm between pairs $(S, T)$ with $S \subset[n]$ and $T$ a $\lambda$-tableau, and $\mu$-tableau $T^{\prime}$ where $\mu$ is a partition such that $\lambda \rightrightarrows \mu$. Furthermore, if we let $x^{T}=\prod_{r \in T} x_{r}$, we note that the insertion algorithm at no time creates destroys or changes the numbers occurring in the tableau, only moves them and thus $x^{T} x^{S}=x^{(S \hookrightarrow T)}$.

Therefore,

$$
\begin{aligned}
G_{\lambda}(x) \Pi(x) & =\sum_{\operatorname{sh}(\mathrm{T})=\lambda} \beta^{|T|-|\lambda|} x^{T} \sum_{S \subset[n]} \beta^{|S|} x^{S} \\
& =\sum_{(T, S)} \beta^{|T|+|S|-|\lambda|} x^{(S \hookrightarrow T)} \\
& =\sum_{\lambda \rightrightarrows \mu} \beta^{|\mu / \lambda|} \sum_{\operatorname{sh}\left(\mathrm{T}^{\prime}\right)=\mu} \beta^{\left|T^{\prime}\right|-|\mu|} x^{T^{\prime}} \\
& =\sum_{\lambda \rightrightarrows \mu} \beta^{|\mu / \lambda|} G_{\mu}(x)
\end{aligned}
$$

as required.

This last result provides the values of $c_{\lambda(1)}^{\nu}$ for all partitions $\lambda$ and $\nu$. Later, we shall prove Theorem 6.7 providing a rule describing the general coefficient $c_{\theta \mu}^{\nu}$. This theorem encompasses two special cases which are known thanks to Buch [2], namely that when $\theta$ is a partition, and when $\mu=\phi$, the empty partition. We shall finish off this section by quoting these results. In order to do so however, we first need to introduce the idea of a lattice word.

Definition 3.9. We say that a sequence of positive integers $w=\left(i_{1}, i_{2}, \ldots, i_{l}\right)$ has content $\left(c_{1}, c_{2}, \ldots\right)$ if $c_{j}$ is equal to the number of occurrences of $j$ in $w$. We call $w$ a lattice word if for each $k$, the content of the subsequence $\left(i_{1}, i_{2}, \ldots, i_{k}\right)$ is a partition.

For the case where $\theta=\lambda$, a partition, Buch's result is as follows:

Theorem 3.10. [2] $c_{\lambda \mu}^{\nu}$ is equal to $\beta^{|\nu|-|\lambda|-|\mu|}$ times the number of set-valued tableaux $T$ of shape $\lambda * \mu$ such that $c(T)$ is a lattice word with content $\nu$.

Here, $\lambda * \mu$ is defined to be the skew diagram obtained by adjoining the top right hand corner of $\lambda$ to the bottom left corner of $\mu$ as shown in the diagram below. 


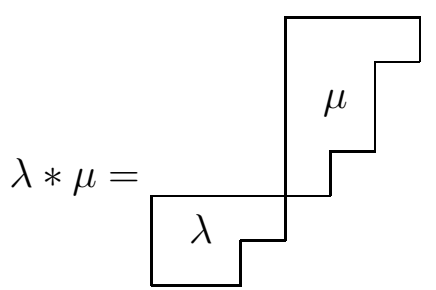

For the case where $\mu=\phi$, the empty partition, Buch's result, expanding the skew Grothendieck polynomial $G_{\theta}(x)$ in the basis $\left\{G_{\lambda}(x) \mid \ell(\lambda) \leq n\right\}$ is as follows:

Theorem 3.11. [2] $c_{\theta \phi}^{\nu}$ is equal to the number of set-valued tableaux of shape $\theta$ such that $c(T)$ is a lattice word with content $\nu$.

\section{The Factorial Grothendieck Polynomials}

Now we are ready to begin our study of the factorial Grothendieck polynomials, the main focus of this paper. Again, we work over an arbitrary field $\mathbb{F}$, and let $\beta$ be an indeterminate over $\mathbb{F}$. In addition to this, we shall also have to introduce a second family of variables as part of the factorial Grothendieck polynomials.

Define the binary operation $\oplus$ (borrowed from [4] and [5]) by

$$
x \oplus y=x+y+\beta x y
$$

and denote the inverse of $\oplus$ by $\ominus$, so we have $\ominus x=\frac{-x}{1+\beta x}$ and $x \ominus y=\frac{x-y}{1+\beta y}$.

\subsection{Definition and basic properties}

Let $\theta$ be a skew diagram, $a=\left(a_{k}\right)_{k \in \mathbb{Z}}$ be a sequence of variables (in the most important case, where $\theta$ is a partition, we only need to consider $\left.\left(a_{k}\right)_{k=1}^{\infty}\right)$. We are now in a position to define the factorial Grothendieck polynomials in $n$ variables $x=\left(x_{1}, x_{2}, \ldots, x_{n}\right)$.

Definition 4.1 (Factorial Grothendieck Polynomials). The factorial Grothendieck polynomial $G_{\theta}(x \mid a)$ is defined to be

$$
G_{\theta}(x \mid a)=\sum_{T} \beta^{|T|-|\theta|} \prod_{\substack{\alpha \in \theta \\ r \in T(\alpha)}} x_{r} \oplus a_{r+c(\alpha)},
$$

recalling that $c(\alpha)$ is the content of the cell $\alpha$, defined by $c(i, j)=j-i$. The summation is taken over all semistandard set-valued $\theta$-tableaux $T$.

\section{$\underline{\text { Remarks }}$}

1. The name factorial Grothendieck polynomial is chosen to stress the analogy with the factorial Schur functions, as mentioned for example (though not explicitly with this name), in variation 6 of MacDonald's theme and variations of Schur functions [12]. The 
factorial Schur functions are obtainable as a specialisation of the factorial Grothendieck polynomials by setting $\beta=0$, though to be truly consistent with the established literature, one should accompany this specialisation with the transformation $a \mapsto-a$.

2. Setting $\theta=\phi$, the empty partition, we get $G_{\phi}(x \mid a)=1$.

3. The $\beta$ can be seen to play the role of marking the degree. For if we assign a degree of -1 to $\beta$, where $x$ and $a$ each have degree 1 , then $G_{\theta}(x \mid a)$ becomes homogenous of degree $|\theta|$.

4. If we set $a=0$, then we recover the ordinary Grothendieck polynomials through specialisation.

5. If $\lambda$ is a partition with $\ell(\lambda)>n$, then it is impossible to fill the first column of $\lambda$ to form a semistandard tableau, so $G_{\lambda}(x \mid a)=0$. Hence we tend to work only with partitions of at most $n$ parts.

6. In a similar vein to the connection between factorial Schur functions and double Schubert polynomials, as pointed out by Lascoux [9], there exists a relationship between these factorial Grothendieck polynomials and the double Grothendieck polynomials discussed for example in [2], amongst other places. The final two sections of this paper work towards proving such a result, culminating in Theorem 8.7, which provides a succinct relationship between these two different types of Grothendieck polynomials.

Example 4.2. Let us calculate $G_{(1)}(x \mid a)$. Here we use $x \oplus a$ to represent the sequence $\left(x_{1} \oplus a_{1}, x_{2} \oplus a_{2}, \ldots, x_{n} \oplus a_{n}\right)$.

Similarly to the calculation of $G_{(1)}(x)$, we can have any nonempty subset of $[n]$ in the one box of $T$, so we have

$$
\begin{aligned}
G_{(1)}(x \mid a) & =\sum_{\substack{S \subset[n] \\
S \neq \phi}} \beta^{|S|-1} \prod_{i \in S} x_{i} \oplus a_{i}=\sum_{j=1}^{n} \beta^{j-1} \sum_{\substack{S \subset[n] \\
|S|=j}} \prod_{i \in S} x_{i} \oplus a_{i} \\
& =\sum_{j=1}^{n} \beta^{j-1} e_{j}(x \oplus a),
\end{aligned}
$$

where the $e_{j}$ are the elementary symmetric functions. Hence,

$$
1+\beta G_{(1)}(x \mid a)=\sum_{j=0}^{n} \beta^{j} e_{j}(x \oplus a)=\prod_{j=1}^{n}\left(1+\beta\left(x_{j} \oplus a_{j}\right)\right)=\Pi(x) \Pi(a) .
$$

Theorem 4.3. The factorial Grothendieck polynomials are symmetric in $x_{1}, x_{2}, \ldots, x_{n}$.

Proof. (This proof is a generalisation of a standard argument, for example as appears in [17, Prop 4.4.2].) The symmetric group $S_{n}$ acts on the ring of polynomials in $n$ variables $x_{1}, x_{2}, \ldots, x_{n}$ by permuting variables: $\pi P\left(x_{1}, \ldots, x_{n}\right)=P\left(x_{\pi(1)}, \ldots, x_{\pi(n)}\right)$ for $\pi \in S_{n}$. Since the adjacent transpositions $(i, i+1)$ generate $S_{n}$, to show that $G_{\theta}(x \mid a)$ is symmetric it suffices to show that $G_{\theta}(x \mid a)$ is stable under interchanging $x_{i}$ and $x_{i+1}$. We consider marked semistandard tableaux, with an entry $j$ marked in one of three ways: 
1. first marking - $j$ - corresponding to taking the $x$ term from $x \oplus a$.

2. second marking $-j^{*}$ - taking the $a$ term from $x \oplus a$.

3. third marking $-j j^{*}$ - taking the $\beta x a$ term from $x \oplus a$.

Then we can write $G_{\theta}(x \mid a)$ as a sum over marked tableaux, where each marked tableau $T$ contributes the monomial

$$
w(T)=\beta^{|T|-|\theta|} \prod_{r \text { unstarred }} x_{r} \prod_{r \text { starred }} a_{r+c(\alpha)}
$$

to the sum $G_{\theta}(x \mid a)=\sum_{T} w(T)$.

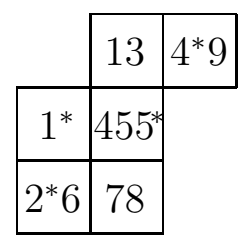

As an example, if $T$ is the above tableau, then we have

$$
w(T)=\beta^{7} x_{9} a_{6} x_{3} a_{2} x_{5} a_{5} x_{4} x_{8} x_{7} a_{0} x_{6} a_{0} .
$$

Note that there is no ambiguity between first and third, or second and third markings, since the same number cannot occur twice in the same cell.

We now find a bijection $T \rightarrow T^{\prime}$ between marked tableaux such that $(i, i+1) w(T)=$ $w\left(T^{\prime}\right)$.

Given $T$, we construct $T^{\prime}$ as follows:

All entries not $i$ or $i+1$ remain unchanged.

If $i$ and $i+1$ appear in the same column, we swap their markings. An example with $i=2$ is the following:

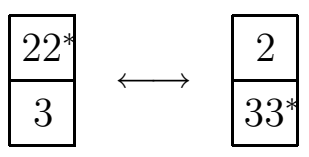

All other occurrences of $i$ and $i+1$ are called free, and we deal with them one row at a time, independently of each other.

Suppose that there are $a$ free $i$ 's and $b$ free $i+1$ 's in a row. Here we are not counting starred markings and also not distinguishing between an unstarred number in the first marking and in the third marking. If $a=b$, the row remains unchanged. Now let us assume that $a>b$. (The $a<b$ case can proceed similarly, or alternatively and equivalently can be defined to be the inverse of the $a>b$ case.)

Consider those cells from the $(b+1)$-th free $i$ to the $a$-th free $i$ inclusive, and call these cells $L$. If the rightmost box of $L$ contains an unstarred $i+1$, we extend $L$ to the left to start at the $b$-th free $i$. We leave boxes outside of $L$ unchanged and modify boxes in $L \subset T$ to form $L^{\prime} \subset T^{\prime}$ as follows: 
1. For each second marking $i^{*}$ in $L$, not in the leftmost box of $L$, replace it by an $(i+1)^{*}$ in $L^{\prime}$ one box to the left. Similarly, for each third marking $i i^{*}$ in $L$, not in the leftmost box of $L$, we replace it by an $(i+1)(i+1)^{*}$ in $L^{\prime}$ one box to the left.

2. Place an $i+1$ in any cells of $L^{\prime}$ which are still empty.

3. Any $i^{*}$ in the leftmost box of $L$ or an $(i+1)^{*}$ in the rightmost box of $L^{\prime}$ is left unchanged.

4. If there is an $i+1$ in the last square of $L$, place an $i$ in the first square of $L^{\prime}$.

To illustrate this more clearly, we provide now an example of the bijection between free rows (note in this example, $i=2, a=3, b=1$, and $L$ runs from the third to the sixth cells in the row inclusive).

\begin{tabular}{|l|l|l|l|l|l|l|}
\hline $2^{*}$ & 2 & $22^{*}$ & $2^{*}$ & 2 & $23^{*}$ & 3 \\
\hline
\end{tabular}$\longleftrightarrow$\begin{tabular}{|l|l|l|l|l|l|l|}
\hline $2^{*}$ & 2 & $2^{*} 3^{*}$ & 3 & 3 & $33^{*}$ & 3 \\
\hline
\end{tabular}

From the structure of the construction of the map $T \mapsto T^{\prime}$, we can easily see that it is an involution, so thus is bijective, and furthermore except that the number of $i$ 's and the number of $(i+1)$ 's is reversed, weights are preserved, in the sense that we have the desired equation $(i, i+1) w(T)=w\left(T^{\prime}\right)$. Thus we have

$$
(i, i+1) G_{\theta}(x \mid a)=\sum_{T}(i, i+1) w(T)=\sum_{T^{\prime}} w\left(T^{\prime}\right)=G_{\theta}(x \mid a)
$$

as required, so the proof is complete.

Given a partition $\lambda=\left(\lambda_{1}, \lambda_{1}, \ldots, \lambda_{n}\right)$, define the sequence $a_{\lambda}=\left(\left(a_{\lambda}\right)_{1},\left(a_{\lambda}\right)_{2}, \ldots,\left(a_{\lambda}\right)_{n}\right)$ by $\left(a_{\lambda}\right)_{i}=\ominus a_{n+1-i+\lambda_{i}}=\frac{-a_{n+1-i+\lambda_{i}}}{1+\beta a_{n+1-i+\lambda_{i}}}$

Theorem 4.4 (Vanishing Theorem). Suppose $\lambda$ and $\mu$ are partitions with $\ell(\lambda) \leq n$. Then

$$
\begin{gathered}
G_{\lambda}\left(a_{\mu} \mid a\right)=0 \text { if } \lambda \not \subset \mu \\
G_{\lambda}\left(a_{\lambda} \mid a\right) \neq 0
\end{gathered}
$$

Proof. (This argument is borrowed from Okounkov's paper [16] and is included here for completeness and its importance.)

Since $G_{\lambda}(x \mid a)$ is symmetric in $x$, we can replace the sequence $\left(x_{1}, x_{2}, \ldots, x_{n}\right)$ by $\left(x_{n}, x_{n-1}, \ldots, x_{1}\right)$ in $(4.1)$ to obtain

$$
G_{\lambda}(x \mid a)=\sum_{T} \beta^{|T|-|\lambda|} \prod_{\substack{\alpha \in \lambda \\ r \in T(\alpha)}} x_{n+1-r} \oplus a_{r+c(\alpha)} .
$$

Thus we have

$$
\begin{aligned}
G_{\lambda}\left(a_{\mu} \mid a\right) & =\sum_{T} \beta^{|T|-|\lambda|} x^{T} \\
\text { where } \quad x^{T} & =\prod_{\substack{\alpha \in \lambda \\
r \in T(\alpha)}} \frac{a_{r+c(\alpha)}-a_{r+\mu_{n+1-r}}}{1+\beta a_{r+\mu_{n+1-r}}} .
\end{aligned}
$$

In order to continue, we need the following proposition. 
Proposition 4.5. $x^{T} \neq 0$ if and only if $T(i, j) \geq n+i-\mu_{j}^{\prime}$ for all $(i, j) \in \lambda$.

Proof. (As well as representing a set, sometimes we write $T(\alpha)$ here and treat it like an integer, to do so means that the result holds for any element of $T(\alpha)$.)

We have

$$
\begin{aligned}
x^{T} & \neq 0 \\
\Longleftrightarrow \mu_{n+1-T(\alpha)} & \neq c(\alpha) \quad \forall \alpha
\end{aligned}
$$

Assuming that this holds, we shall now prove by induction on $j$ that $\mu_{n+1-T(1, j)} \geq j$. For $j=1$, by $(4.3), \mu_{n+1-T(1,1)} \neq 0$ so $\mu_{n+1-T(1,1)} \geq 1$ as it is a non-negative integer.

Now suppose $j>1$ and $\mu_{n+1-T(1, j-1)} \geq j-1$.

As $T(1, j) \geq T(1, j-1), \mu_{n+1-T(1, j)} \geq \mu_{n+1-T(1, j-1)}$.

But by $(4.3), \mu_{n+1-T(1, j)} \neq j-1$ so it must be that $\mu_{n+1-T(1, j)} \geq j$ as required and the induction is complete.

Therefore $n+1-T(1, j) \leq \mu_{j}^{\prime}$, i.e. $T(1, j) \geq n+1-\mu_{j}^{\prime}$. Since $T(i+1, j)>T(i, j)$, a straightforward induction on $i$ gives $T(i, j) \geq n+i-\mu_{j}^{\prime}$.

Now suppose that $T(i, j) \geq n+i-\mu_{j}^{\prime}$ for all $(i, j) \in \lambda$. Equivalently this can be written as $n+i-T(i, j) \leq \mu_{j}^{\prime}$, which gives us the chain of inequalities $\mu_{n+1-T(i, j)} \geq \mu_{n+i-T(i, j)} \geq$ $\mu_{\mu_{j}^{\prime}} \geq j>j-i$. In particular, this shows $\mu_{n+1-T(\alpha)} \neq c(\alpha)$, so $x^{T} \neq 0$ as required and the proof of the proposition is complete.

Now we return to proving the vanishing theorem and apply the condition that $T(i, j) \leq$ $n$ to the above proposition. If $G_{\lambda}\left(a_{\mu} \mid a\right) \neq 0$, then we have:

$$
\lambda_{j}^{\prime} \geq i \Longrightarrow(i, j) \in \lambda \Longrightarrow n+i-\mu_{j}^{\prime} \leq n \Longrightarrow \mu_{j}^{\prime} \geq i .
$$

Thus $\lambda_{j}^{\prime} \leq \mu_{j}^{\prime}$ for all $j$, so $\lambda \subset \mu$ and the first part of the vanishing theorem is proven.

In the case $\lambda=\mu$, equality must hold everywhere, so there is a unique $\lambda$-tableau $T$ for which $x^{T} \neq 0$, namely that with $T(i, j)=n+i-\lambda_{j}^{\prime}$ for all $(i, j) \in T$. Hence $G_{\lambda}\left(a_{\lambda} \mid a\right) \neq 0$.

From the above, we can write an explicit formula for $G_{\lambda}\left(a_{\lambda} \mid a\right)$. After making the change $i \rightarrow 1+\lambda_{j}^{\prime}-i$ to neaten up the result, we get:

$$
G_{\lambda}\left(a_{\lambda} \mid a\right)=\prod_{(i, j) \in \lambda} \frac{a_{n+j-\lambda_{j}^{\prime}}-a_{\lambda_{i}+n-i+1}}{1+\beta a_{\lambda_{i}+n-i+1}} .
$$

We pause to introduce a space utilised in a couple of subsequent proofs. Let $L_{k}$ denote the subspace of $\Lambda_{n} \otimes \mathbb{F}(\beta, a)$ spanned by the monomial symmetric functions $\left\{m_{\lambda} \mid \lambda \subset k^{n}\right\}$.

Theorem 4.6. $\left\{G_{\lambda}(x \mid a)\right\}$ form a basis in $\Lambda_{n} \otimes_{\mathbb{Z}} \mathbb{F}(\beta, a)$ as $\lambda$ runs over all partitions with $\ell(\lambda) \leq n$. 
Proof. If $\mu \subset k^{n}$, then $G_{\mu}(x \mid a) \in L_{k}$, for a number $i$ in a $\mu$-tableau $T$ can appear at most once in each column, and hence at most $k$ times overall so the exponent of $x_{i}$ in $G_{\mu}(x \mid a)$ is at most $k$.

Let $\rho_{1}<\rho_{2}<\cdots<\rho_{l}$ be a fixed ordering of the $l=\left(\begin{array}{c}n+k \\ k\end{array}\right)$ partitions contained in $\left(k^{n}\right)$ which is a refinement of the dominance ordering. Define the matrix $D_{k}$ by

$$
\left(\begin{array}{c}
G_{\rho_{1}}(x \mid a) \\
\vdots \\
G_{\rho_{l}}(x \mid a)
\end{array}\right)=D_{k}\left(\begin{array}{c}
m_{\rho_{1}}(x) \\
\vdots \\
m_{\rho_{l}}(x)
\end{array}\right)
$$

which is possible since $\left\{m_{\lambda} \mid \lambda \subset k^{n}\right\}$ forms a basis of $L_{k}$.

Let $d_{k}=d_{k}(\beta, a)=\operatorname{det} D_{k}$.

From the definition of $G_{\lambda}(x \mid a)$, we see that every entry of $D_{k}$ is a polynomial in $\beta$ and $a$ and hence the same is true of $d_{k}$.

If we specialise to the case $\beta=0, a=0$, then $D_{k}$ becomes the transition matrix from the monomial symmetric functions to the classical Schur functions. In [13, Ch 1, 6.5], this transition matrix is shown to be lower triangular, with 1's along the main diagonal, so has determinant 1 and thus $d_{k}(0,0)=1$. Hence $d(\beta, a)$ is not identically zero, so $D_{k}$ is invertible and thus $\left\{G_{\lambda}(x \mid a) \mid \lambda \subset k^{n}\right\}$ is a basis of $L_{k}$.

As $L_{0} \subset L_{1} \subset L_{2} \subset \cdots$ and $\cup_{k=0}^{\infty} L_{k}=\Lambda_{n} \otimes \mathbb{F}(\beta, a),\left\{G_{\lambda}(x \mid a) \mid \ell(\lambda) \leq n\right\}$ forms a basis for $\Lambda_{n} \otimes \mathbb{F}(\beta, a)$.

\subsection{Analysis of poles}

In this section, we do not make any use of skew diagrams, so only need to deal with the sequence of variables $\left(a_{k}\right)_{k=1}^{\infty}$.

Suppose $P(x) \in \Lambda_{n} \otimes \mathbb{F}[\beta, a]$, and suppose we expand $P(x)$ in the basis of factorial Grothendieck polynomials $G_{\lambda}(x \mid a)$.

$$
P(x)=\sum_{\lambda} d_{\lambda} G_{\lambda}(x \mid a)
$$

The coefficients $d_{\lambda}$ can be written as a quotient of coprime polynomials in $\beta$ and $a$ : $d_{\lambda}=f_{\lambda} / g_{\lambda}$.

Lemma 4.7. The only possible irreducible factors of $g_{\lambda}$ are of the form $1+\beta a_{i}$ for some $i>0$.

Proof. First, fix a $k$ such that $P(x) \in L_{k}$. If we first expand $P(x)$ in the basis of monomial symmetric functions $m_{\lambda}$, we find that the coefficients are all polynomials in $\beta$ and $a$. Using (4.4) and Cramer's rule to subsequently determine the $d_{\lambda}$, we find that the denominators $g_{\lambda}$ must all divide $d_{k}$.

Setting $x=a_{\mu}$ in (4.5) and applying the vanishing theorem gives

$$
d_{\mu}=\frac{1}{G_{\mu}\left(a_{\mu} \mid a\right)}\left(P\left(a_{\mu}\right)-\sum_{\rho \subsetneq \mu} d_{\rho} G_{\rho}\left(a_{\mu} \mid a\right)\right) .
$$


This provides a recurrence from which the coefficients $d_{\lambda}$ can be computed inductively using inclusion ordering. From such an induction, we can conclude that the only possible irreducible factors of the denominators $g_{\lambda}$ are of the form $1+\beta a_{i}$ (from poles of $G_{\rho}\left(a_{\mu} \mid a\right)$ ) or $a_{i}-a_{j}$ (from zeros of $G_{\mu}\left(a_{\mu} \mid a\right)$ ). If the latter occurs, then $g_{\lambda}(0,0)=0$, contradicting $d_{k}(0,0)=1$ as $g_{\lambda} \mid d_{k}$. Thus the only possible irreducible factors of denominators $g_{\lambda}$ are of the form $1+\beta a_{i}(i>0)$.

In fact we can prove that the only possible irreducible factors of $d_{k}(\beta, a)$ are of the form $1+\beta a_{i}$ for some $i$. For if $f$ is irreducible and $f$ divides $d_{k}$, then working over the integral domain $\mathbb{F}[\beta][a] /(f)$, where $(f)$ is the ideal generated by $f$, we have that the determinant of the transition matrix from the monomial symmetric functions to the factorial Grothendieck polynomials is zero. Hence the factorial Grothendieck polynomials are linearly dependent. So there exist $c_{\lambda} \in \mathbb{F}[\beta][a] /(f)$ not all zero such that $\sum_{\lambda} c_{\lambda} G_{\lambda}(x \mid a)=0$. If $b_{\lambda} \in \mathbb{F}[\beta][a]$ is such that $c_{\lambda}=b_{\lambda}+(f)$ then $\sum_{\lambda} b_{\lambda} G_{\lambda}(x \mid a)=f g$ for some $g \in \mathbb{F}[\beta][a][x]$ and not all $b_{\lambda}$ are divisible by $f$. Then $g=\sum_{\lambda} \frac{b_{\lambda}}{f} G_{\lambda}$ and since not all $b_{\lambda}$ are divisible by $f, f$ can appear as a denominator of an expansion of the form (4.5), and hence from our above result concerning such denominators, $f$ must be of the form $1+\beta a_{i}$ for some $i$.

In the subsequent section, we shall prove the following formula, which shows that for all $i>0,1+\beta a_{i}$ can appear as a factor of a denominator in an expansion of the form (4.5), and hence divides $d_{k}$ for large enough $k$.

\section{Proposition 4.8.}

$$
G_{\lambda}(x \mid a) \Pi(x)=\Pi\left(a_{\lambda}\right) \sum_{\lambda \rightrightarrows \mu} \beta^{|\mu / \lambda|} G_{\mu}(x \mid a)
$$

Once this formula is proven, we have the stronger result.

Theorem 4.9. The specialisation of $\left\{G_{\lambda}(x \mid a) \mid \ell(\lambda) \leq n\right\}$ under an evaluation homomorphism $\mathbb{F}[\beta, a] \rightarrow \mathbb{F}$ forms a basis of $\Lambda_{n} \otimes \mathbb{F}$ if and only if $a_{i} \beta \neq-1$ for all $i$.

Note that this also includes the important case of the ordinary Grothendieck polynomials via the specialisation $a=0$.

\section{A Recurrence for the Coefficients}

\subsection{Proof of Proposition 4.8}

Define coefficients $c_{\lambda}^{\mu}=c_{\lambda}^{\mu}(\beta, a)$ by

$$
\frac{G_{\lambda}(x \mid a) \Pi(x)}{\Pi\left(a_{\lambda}\right)}=\sum_{\mu} \beta^{|\mu|-|\lambda|} c_{\lambda}^{\mu} G_{\mu}(x \mid a) .
$$

These are well defined since the factorial Grothendieck polynomials are known to form a basis. (Theorem 4.6.) 
First, consider (5.1) with $x$ and $a$ replaced by $x / \beta$ and $a / \beta$ respectively:

$$
\frac{\beta^{|\lambda|} G_{\lambda}\left(\frac{x}{\beta} \mid \frac{a}{\beta}\right) \Pi\left(\frac{x}{\beta}\right)}{\Pi\left(\frac{a_{\lambda}}{\beta}\right)}=\sum_{\mu} c_{\lambda}^{\mu}\left(\beta, \frac{a}{\beta}\right) \beta^{|\mu|} G_{\mu}\left(\frac{x}{\beta} \mid \frac{a}{\beta}\right) .
$$

Terms of the form $\beta^{|\nu|} G_{\nu}\left(\frac{x}{\beta} \mid \frac{a}{\beta}\right)$ and $\Pi\left(\frac{y}{\beta}\right)$ are both independent of $\beta$. Hence $c_{\lambda}^{\mu}\left(\beta, \frac{a}{\beta}\right)$ is also independent of $\beta$. As we already know $c_{\lambda}^{\mu}$ is a rational function of $\beta$ and $a$, this last piece of information tells us that in fact $c_{\lambda}^{\mu}$ is a rational function of $\beta a_{1}, \beta a_{2}, \ldots$

Setting $x=a_{\mu}$ in (5.1) and applying the vanishing theorem gives:

$$
c_{\lambda}^{\mu}=\frac{1}{\beta^{|\mu|-|\lambda|} G_{\mu}\left(a_{\mu} \mid a\right)}\left(\frac{G_{\lambda}\left(a_{\mu} \mid a\right) \Pi\left(a_{\mu}\right)}{\Pi\left(a_{\lambda}\right)}-\sum_{\rho \subsetneq \mu} \beta^{|\rho|-|\lambda|} c_{\lambda}^{\rho} G_{\rho}\left(a_{\mu} \mid a\right)\right)
$$

from which we compute the coefficients $c_{\lambda}^{\mu}$ inductively on $\mu$.

If $\rho$ is a minimal partition with respect to containment order for which $c_{\lambda}^{\rho} \neq 0$, then this gives $G_{\lambda}\left(a_{\rho} \mid a\right) \neq 0$, so by the vanishing theorem, $\lambda \subset \rho$. So we may rewrite our sum in (5.2) as a sum over $\lambda \subset \rho \subsetneq \mu$.

We shall now prove by induction on $\mu$ that $c_{\lambda}^{\mu} \in \mathbb{F}[\beta, a]$. So suppose that $c_{\lambda}^{\rho} \in \mathbb{F}[\beta, a]$ for all $\rho \subsetneq \mu$.

From (5.2), we find that the following list gives all possibilities for poles of $c_{\lambda}^{\mu}$ :

1. zeros of $\beta^{|\mu|-|\lambda|} G_{\mu}\left(a_{\mu} \mid a\right)$.

2. poles of $\beta^{|\rho|-|\lambda|} c_{\lambda}^{\rho} G_{\rho}\left(a_{\mu} \mid a\right)$, where $\lambda \subset \rho \subsetneq \mu$.

3. poles of $G_{\lambda}\left(a_{\mu} \mid a\right) \Pi\left(a_{\mu}\right) \Pi\left(a_{\lambda}\right)^{-1}$.

1. Zeros of $\beta^{|\mu|-|\lambda|} G_{\mu}\left(a_{\mu} \mid a\right)$ are of the form $\beta$ or $a_{i}-a_{j}$. However $G_{\lambda}(x \mid a) \Pi(x) \Pi\left(a_{\lambda}\right)^{-1} \in$ $\Lambda_{n} \otimes \mathbb{F}[\beta, a]$ since $\Pi\left(a_{\lambda}\right)^{-1}=\prod_{i=1}^{n}\left(1+\beta a_{n+1-i+\lambda_{i}}\right)$, so by Lemma 4.7 , the poles of $\beta^{|\mu|-|\lambda|} c_{\lambda}^{\mu}$ can only have irreducible factors of the form $1+\beta a_{i}$. This leaves open the possibility that $\beta$ could be a pole of $c_{\lambda}^{\mu}$. If this were the case, since $c_{\lambda}^{\mu}$ is a rational function of $\beta a, c_{\lambda}^{\mu}$ would also have to contain a pole which vanishes at $a=0$, contradicting our general result concerning poles.

2. A pole of $\beta^{|\rho|-|\lambda|}$ cannot be a pole of $c_{\lambda}^{\mu}$ by the argument above. By inductive assumption, there do not exist any poles of $c_{\lambda}^{\rho}$, where $\rho \subsetneq \mu$. Now write $G_{\rho}\left(a_{\mu} \mid a\right)=$ $\sum_{T} \beta^{|T|-|\rho|} x^{T}$ as per (4.2). By our proposition, if $x^{T} \neq 0$, then $T$ can have at most the entries $n+1-\mu_{j}^{\prime}, n+2-\mu_{j}^{\prime}, \ldots, n$ in the $j$-th column. This maximal set of entries are exactly the entries of the unique tableau $T$ which contributes a non-zero amount to $G_{\mu}\left(a_{\mu} \mid a\right)$. Hence the pole of $x^{T}$ is at most that of $G_{\mu}\left(a_{\mu} \mid a\right)$, so since we divide by $G_{\mu}\left(a_{\mu} \mid a\right)$, this gives no contributions to poles of $c_{\lambda}^{\mu}$.

3. Write $G_{\lambda}\left(a_{\mu} \mid a\right)=\sum_{T} \beta^{|T|-|\lambda|} x^{T}$ as per (4.2). Then we have

$$
G_{\lambda}\left(a_{\mu} \mid a\right) \frac{\Pi\left(a_{\mu}\right)}{\Pi\left(a_{\lambda}\right)}=\sum_{T} \beta^{|T|-|\lambda|} x^{T} \frac{\Pi\left(a_{\mu}\right)}{\Pi\left(a_{\lambda}\right)} .
$$


Suppose that $c_{\lambda}^{\mu}$ has a factor $1+\beta a_{k}$ in its denominator. Then $1+\beta a_{k}$ is a pole of $x^{T}$ or $\Pi\left(a_{\mu}\right)$ so is of the form $1+\beta a_{i+\mu_{n+1-i}}$ for some $i \in[n]$.

We only need to consider those tableaux $T$ for which the multiplicity of the factor $1+\beta a_{i+\mu_{n+1-i}}$ in the denominator of $x^{T} \Pi\left(a_{\mu}\right)$ is strictly greater than the corresponding multiplicity in $G_{\mu}\left(a_{\mu} \mid a\right)$. From the argument in Case 2, we know that $x^{T}$ has a pole at most that of $G_{\mu}\left(a_{\mu} \mid a\right)$.

If $\mu_{n+1-i}=\lambda_{n+1-i}$, then there will be a corresponding factor $1+\beta a_{k}$ in $\Pi\left(a_{\lambda}\right)$ cancelling that from $\Pi\left(a_{\mu}\right)$ ensuring that the multiplicity of $1+\beta a_{k}$ in the denominator of $x^{T} \Pi\left(a_{\mu}\right) \Pi\left(a_{\lambda}\right)^{-1}$ is not greater than that in $G_{\lambda}\left(a_{\mu} \mid a\right)$, as required.

Now we may suppose $\mu_{n+1-i} \neq \lambda_{n+1-i}$. We also must have $G_{\lambda}\left(a_{\mu} \mid a\right) \neq 0$, so $\mu \supset \lambda$, and thus $\mu_{n+1-i}>\lambda_{n+1-i}$.

Factors of $1+\beta a_{k}$ in the denominator of $x^{T}$ are in one-to-one correspondence with occurrences of the entry $i$ in $T$, so we only need to consider those $T$ with a maximal possible occurrence of $i$ as given by Proposition 4.5.

Consider the $\mu_{n+1-i}$-th column of our $\lambda$-tableau $T$, and call it $C$.

As $\left(n+1-i, \mu_{n+1-i}+1\right) \notin \mu, \mu_{\left(\mu_{n+1-i}+1\right)}^{\prime} \leq n-i$ and thus $T\left(1, \mu_{n+1-i}+1\right)>i$. Hence, there cannot be any $i$ 's to the right of $C$. Also, $n+1-\mu_{\mu_{n+1-i}}^{\prime} \geq i$, so since $T$ contains the maximal possible number of $i$ 's, it must contain an entry $i$ in the $\mu_{n+1-i}$-th column.

Let $j$ be the largest index for which $T\left(j, \mu_{n+1-i}\right)$ contains an entry less than $i+j$. Note $j$ must exist as there must exist an $i$ in this column.

Pair the two tableaux $T$ and $T^{\prime}$, identical in all respects except that $T^{\prime}$ contains an $i+j$ in the $j$-th row of $C$ and $T$ does not. Note that these will both be semistandard since we have the inequalities $T\left(j, \mu_{n+1-i}+1\right) \geq i+j$ (by Proposition 4.5) and $T\left(j+1, \mu_{n+1-i}\right)>i+j$ (by maximality of $j$ ) while $T\left(j, \mu_{n+1-i}\right)$ already contains an entry less than $i+j$.

We now calculate:

$$
\begin{aligned}
\beta^{|T|-|\lambda|} x^{T}+\beta^{\left|T^{\prime}\right|-|\lambda|} x^{T^{\prime}} & =\beta^{|T|-|\lambda|} x^{T}\left(1+\beta\left(\left(a_{\mu}\right)_{n+1-(i+j)}\right) \oplus a_{i+j+c\left(j, \mu_{n+1-i}\right)}\right) \\
& =\beta^{|T|-|\lambda|} x^{T} \frac{1+\beta a_{i+\mu_{n+1-i}}}{1+\beta a_{i+j-\mu_{n+1-(i+j)}}}
\end{aligned}
$$

By pairing our tableaux in this way and recovering an extra factor $1+\beta a_{k}$ in the numerator, we see that the total pole for the factor $1+\beta a_{k}$ is at most that of $G_{\mu}\left(a_{\mu} \mid a\right)$ as required, so $c_{\lambda}^{\mu}$ cannot have any poles, since all possible cases have now been considered.

Thus $c_{\lambda}^{\mu}$ is a polynomial in $\beta$ and $a$ (as we already know it is a rational function of $\beta$ and $a$ ). We now compute the degree of $c_{\lambda}^{\mu}$, considered as a polynomial in $\beta$, and show by induction on $\mu$ that $\operatorname{deg}_{\beta} c_{\lambda}^{\mu} \leq 0$.

We use equation (5.2) and calculate the degree of each of its constituent terms.

\begin{tabular}{|c|c|}
\hline term & degree \\
\hline$\beta^{|\mu|-|\lambda|} G_{\mu}\left(a_{\mu} \mid a\right)$ & $|\mu|-|\lambda|-|\mu|=-|\lambda|$ \\
$G_{\lambda}\left(a_{\mu} \mid a\right)$ & $\leq-|\lambda|$ \\
$\Pi\left(a_{\mu}\right) \Pi\left(a_{\lambda}\right)^{-1}$ & 0 \\
$\beta^{|\rho|-|\lambda|} c_{\lambda}^{\rho} G_{\rho}\left(a_{\mu} \mid a\right)$ & $\leq|\rho|-|\lambda|+0-|\rho|=-|\lambda|$ \\
\hline
\end{tabular}


Here we use the fact that $x^{T}$ has degree $-|T|$ and the inductive assumption for $\rho \subsetneq \mu$.

Now placing this into (5.2) we arrive at the inequality $\operatorname{deg} c_{\lambda}^{\mu} \leq-|\lambda|+|\lambda|=0$ as required.

Being a polynomial in $\beta a_{1}, \beta a_{2}, \ldots$ of degree at most zero in $\beta, c_{\lambda}^{\mu}$ must be constant, that is independent of $\beta$ and $a$. Thus we can calculate the values of $c_{\lambda}^{\mu}$ by specialisation to the ordinary Grothendieck polynomials with $a=0$. From Proposition 3.8, we know the value of $c_{\lambda}^{\mu}(\beta, 0)$ and thus,

$$
c_{\lambda}^{\mu}(\beta, a)=c_{\lambda}^{\mu}(\beta, 0)= \begin{cases}1, & \text { if } \lambda \rightrightarrows \mu \\ 0, & \text { otherwise }\end{cases}
$$

so Proposition 4.8 is proven, as required.

\subsection{The recurrence relation}

Suppose $\nu$ is a partition of length at most $n, \mu$ is a partition, $\theta$ is a skew diagram and $P(x)$ is a fixed symmetric polynomial in $x_{1}, x_{2} \ldots x_{n}$ with coefficients in $\mathbb{F}(\beta, a)$. Then define the coefficients $g_{\mu}^{\nu}=g_{\mu}^{\nu}(P) \in \mathbb{F}(\beta, a)$ by the formula

$$
P(x) G_{\mu}(x \mid a)=\sum_{\nu} g_{\mu}^{\nu} G_{\nu}(x \mid a) .
$$

Theorem 4.6 ensures that these coefficients are well defined.

In the important special case where $P(x)=G_{\theta}(x \mid b)$ with $b=\left(b_{i}\right)_{i \in \mathbb{Z}}$ a second doubly infinite sequence of variables, we use the notation $g_{\theta \mu}^{\nu}=g_{\theta \mu}^{\nu}(a, b)$ for $g_{\mu}^{\nu}\left(G_{\theta}(x \mid b)\right)$.

Proposition 5.1. The coefficients $g_{\mu}^{\nu}$ satisfy the following recurrence:

$$
g_{\mu}^{\nu}=\frac{1}{\Pi\left(a_{\nu}\right)-\Pi\left(a_{\mu}\right)}\left(\sum_{\mu \rightrightarrows^{*} \lambda} \Pi\left(a_{\mu}\right) \beta^{|\lambda / \mu|} g_{\lambda}^{\nu}-\sum_{\eta \rightrightarrows^{*} \nu} \Pi\left(a_{\eta}\right) \beta^{|\nu / \eta|} g_{\mu}^{\eta}\right),
$$

with boundary conditions

$$
\begin{aligned}
\text { (i) } & g_{\mu}^{\nu} & =0 \quad \text { unless } \quad \mu \subset \nu, \\
\text { (ii) } & g_{\lambda}^{\lambda} & =P\left(a_{\lambda}\right) .
\end{aligned}
$$

This is indeed a recurrence, for it enables the coefficients $g_{\mu}^{\nu}$ to be computed recursively by induction on $|\nu / \mu|$.

Proof. Applying Proposition 4.8 to

$$
P(x) G_{\mu}(x \mid a) \Pi(x)=\sum_{\eta} g_{\mu}^{\eta} G_{\eta}(x \mid a) \Pi(x)
$$

yields the following:

$$
P(x) \Pi\left(a_{\mu}\right) \sum_{\mu \rightrightarrows \lambda} \beta^{|\lambda / \mu|} G_{\lambda}(x \mid a)=\sum_{\eta} g_{\mu}^{\eta} \Pi\left(a_{\eta}\right) \sum_{\eta \rightrightarrows \nu} \beta^{|\nu / \eta|} G_{\nu}(x \mid a) .
$$


If we now combine this with (5.3) we obtain the identity

$$
\Pi\left(a_{\mu}\right) \sum_{\mu \rightrightarrows \lambda} \sum_{\nu} \beta^{|\lambda / \mu|} g_{\lambda}^{\nu} G_{\nu}(x \mid a)=\sum_{\eta} g_{\mu}^{\eta} \Pi\left(a_{\eta}\right) \sum_{\eta \rightrightarrows \nu} \beta^{|\nu / \eta|} G_{\nu}(x \mid a)
$$

We now use the fact that the factorial Grothendieck polynomials $G_{\lambda}(x \mid a)$ form a basis to equate the coefficients of $G_{\nu}(x \mid a)$, giving

$$
\Pi\left(a_{\mu}\right) \sum_{\mu \rightrightarrows \lambda} \beta^{|\lambda / \mu|} g_{\lambda}^{\nu}=\sum_{\eta \rightrightarrows \nu} \Pi\left(a_{\eta}\right) \beta^{|\nu / \eta|} g_{\mu}^{\eta}
$$

which rearranges to the quoted form of the recurrence.

For the boundary conditions, suppose that $\rho$ is a minimal partition with respect to containment order such that $g_{\mu}^{\rho} \neq 0$. Substituting $x=a_{\rho}$ in (5.3) gives $G_{\mu}\left(a_{\rho} \mid a\right) P\left(a_{\rho}\right)=$ $g_{\mu}^{\rho} G_{\rho}\left(a_{\rho} \mid a\right)$. If $\mu \not \subset \rho$ then from the vanishing theorem, we get $(i) g_{\mu}^{\rho}=0$, so now we may deal with the $\mu=\rho$ case which gives $(i i) g_{\rho}^{\rho}=P\left(a_{\rho}\right)$ as required.

We now give a general solution to the above recurrence.

For a partition $\lambda$, introduce the notation $\Pi(\lambda)$ to represent $\Pi\left(a_{\lambda}\right)$.

Proposition 5.2. The general solution to the recurrence (5.4) is

$$
g_{\mu}^{\nu}=\beta^{|\nu / \mu|} \sum_{R} \Pi\left(\rho_{0}\right) \Pi\left(\rho_{1}\right) \ldots \Pi\left(\rho_{l-1}\right) \sum_{k=0}^{l} P\left(a_{\rho_{k}}\right) \prod_{\substack{i=0 \\ i \neq k}}^{l} \frac{1}{\Pi\left(\rho_{k}\right)-\Pi\left(\rho_{i}\right)}
$$

where the sum is over all sequences

$$
R: \mu=\rho_{0} \rightrightarrows^{*} \rho_{1} \rightrightarrows^{*} \cdots \rightrightarrows^{*} \rho_{l-1} \rightrightarrows^{*} \rho_{l}=\nu
$$

Proof. We need to show that this proposed solution satisfies both the recurrence relation and the boundary conditions.

That this proposed solution satisfies the boundary conditions is immediate, for if $\mu \not \subset \nu$ there is no such sequence $R$ while if $\mu=\nu$ there is exactly one such sequence, the trivial sequence with $l=0$. 
By induction on $|\nu / \mu|$, we get

$$
\begin{aligned}
& g_{\mu}^{\nu}=\frac{1}{\Pi(\nu)-\Pi(\mu)}\left(\sum_{\mu \rightrightarrows^{*} \lambda} \Pi\left(a_{\mu}\right) \beta^{|\lambda / \mu|} g_{\lambda}^{\nu}-\sum_{\eta \rightrightarrows^{*} \nu} \Pi\left(a_{\eta}\right) \beta^{|\nu / \eta|} g_{\mu}^{\eta}\right) \\
& =\frac{\beta^{|\nu / \mu|}}{\Pi(\nu)-\Pi(\mu)}\left(\sum_{R} \prod_{j=0}^{l-1} \Pi\left(\rho^{(j)}\right) \sum_{k=1}^{l} P\left(a_{\rho_{k}}\right) \prod_{\substack{i=1 \\
i \neq k}}^{l} \frac{1}{\Pi\left(\rho_{k}\right)-\Pi\left(\rho_{i}\right)}\right. \\
& \left.-\sum_{R} \prod_{j=0}^{l-1} \Pi\left(\rho^{(j)}\right) \sum_{k=0}^{l-1} P\left(a_{\rho_{k}}\right) \prod_{\substack{i=0 \\
i \neq k}}^{l-1} \frac{1}{\Pi\left(\rho_{k}\right)-\Pi\left(\rho_{i}\right)}\right) \\
& =\beta^{|\nu / \mu|} \sum_{R} \prod_{j=0}^{l-1} \Pi\left(\rho^{(j)}\right) \sum_{k=0}^{l} \frac{P\left(a_{\rho^{(k)}}\right)\left[\left(\Pi\left(\rho_{k}\right)-\Pi\left(\rho_{0}\right)\right)-\left(\Pi\left(\rho_{k}\right)-\Pi\left(\rho_{l}\right)\right)\right]}{(\Pi(\nu)-\Pi(\mu)) \prod_{\substack{i=0 \\
i \neq k}}^{l} \Pi\left(\rho_{k}\right)-\Pi\left(\rho_{i}\right)} \\
& =\beta^{|\nu / \mu|} \sum_{R} \Pi\left(\rho_{0}\right) \Pi\left(\rho_{1}\right) \ldots \Pi\left(\rho_{l-1}\right) \sum_{k=0}^{l} P\left(a_{\rho_{k}}\right) \prod_{\substack{i=0 \\
i \neq k}}^{l} \frac{1}{\Pi\left(\rho_{k}\right)-\Pi\left(\rho_{i}\right)}
\end{aligned}
$$

as required.

\section{Calculation of the Coefficients}

The general solution to the recurrence appears inadequate, in that it is hard to specialise to the case of ordinary Grothendieck polynomials by setting $a=0$, and nor does it clearly reflect the stringent conditions we have imposed on denominators in Lemma 4.7. So now we turn specifically to the case $P(x)=G_{\theta}(x \mid b)$ and provide an alternative description of the coefficients $g_{\theta \mu}^{\nu}$ with a view to specialising to the ordinary Grothendieck polynomials.

\subsection{Solution where all boxes of $\theta$ are in different columns}

We now provide a solution to the recurrence in the case where $\theta$ does not contain two boxes in the same column. In order to state this result however, we first need to define some more combinatorial objects.

Consider a sequence of partitions

$$
R: \mu=\rho^{(0)} \stackrel{r_{1}}{\longrightarrow} \rho^{(1)} \stackrel{r_{2}}{\longrightarrow} \cdots \stackrel{r_{l-1}}{\longrightarrow} \rho^{(l-1)} \stackrel{r_{l}}{\longrightarrow} \rho^{(l)}=\nu .
$$

Say a semistandard set-valued $\theta$ tableau $T$ is related to $R$ if $T$ contains distinguished entries $r_{1}, r_{2}, \ldots, r_{l}$ in cells $\alpha_{1}, \alpha_{2}, \ldots, \alpha_{l}$ respectively with $\left(r_{1}, \alpha_{1}\right) \prec\left(r_{2}, \alpha_{2}\right) \prec \ldots \prec$ $\left(r_{l}, \alpha_{l}\right)$ where $\prec$ is the ordering defined in Section 2.2. We distinguish these entries by placing a bar over them.

If $\rho^{(0)}, \rho^{(1)}, \ldots, \rho^{(l)}$ are partitions, $r_{1}, r_{2} \ldots, r_{l} \in[n]$, and $T$ is a $\theta$-tableau with distinguished entries $\left(r_{1}, \alpha_{1}\right) \prec \cdots \prec\left(r_{l}, \alpha_{l}\right)$, then we define the function

$$
F_{T}\left(\rho^{(l)}|\cdots| \rho_{r_{2}} \rho^{(1)} \mid \rho_{r_{1}}^{(0)}\right)
$$


to equal the product

$$
\prod_{\substack{\alpha \in \theta \\ r \in T(\alpha) \\ \text { unbarred }}}\left(\left(a_{\rho(r)}\right)_{r} \oplus b_{r+c(\alpha)}\right) \cdot \prod_{i=1}^{l}\left(1+\beta\left(a_{\rho^{(i-1)}}\right)_{r_{i}}\right)\left(1+\beta b_{r_{i}+c\left(\alpha_{i}\right)}\right) .
$$

where $\rho(r)=\rho^{(i)}$ if $r_{i} \prec r \prec r_{i+1}$. In the important case where the $\rho^{(i)}$ and $r_{i}$ form a sequence $R$ of the form (6.1), and $T$ is a semistandard $\theta$-tableau related to $R$, we denote this product by $w(T)$.

Theorem 6.1. For $P(x)=G_{\theta}(x \mid b)$, if $\theta$ does not contain two boxes in the same column, then

$$
g_{\theta \mu}^{\nu}=\sum_{(R, T)} \beta^{|T|-|\theta|} w(T) .
$$

where the sum is over all $\theta$-tableaux $T$ which are related to a sequence $R$ of the form (6.1).

Proof. For $\nu \not \supset \mu$, no such sequences $R$ exist so (6.2) agrees with $g_{\theta \mu}^{\nu}=0$ as required.

For $\nu=\mu$, there is one such sequence $R, \rho^{(0)}=\rho^{(l)}=\mu$, so no barred entries can exist in $T$. The set of tableaux summed over is now exactly the same as the set summed over in the definition of $G_{\theta}(x \mid a)$, and we thus notice that $\sum_{(R, T)} \beta^{|T|-|\theta|} w(T)=G_{\theta}\left(a_{\lambda} \mid b\right)$ while the boundary conditions of the recurrence give $g_{\theta \mu}^{\nu}=G_{\theta}\left(a_{\lambda} \mid b\right)$, agreeing with (6.2) as required.

Now we need to show that our proposed solution satisfies the recurrence. So we suppose (6.2) holds and we have to show that this implies (5.4) holds.

Let $m$ be a non-negative integer. Let $l=|\nu / \mu|$. We now form a set $\mathcal{T}_{m}$ of triples $(k, R, T)$ as follows.

$k$ is an integer from $m$ to $l$ inclusive. $R$ is a sequence

$$
R: \mu=\rho^{(0)} \stackrel{r_{1}}{\longrightarrow} \ldots \stackrel{r_{k-m}}{\longrightarrow} \rho^{(k-m)} \rightrightarrows \rho^{(k)} \stackrel{r_{k+1}}{\longrightarrow} \ldots \stackrel{r_{l}}{\longrightarrow} \rho^{(l)}=\nu .
$$

$T$ is a semistandard set-valued $\theta$-tableau $T$ with entries from $[n]$ such that $T$ contains distinguished entries $r_{1}, r_{2}, \ldots, r_{k-m}, r_{k+1}, \ldots, r_{l}$ with $r_{1} \prec r_{2} \prec \ldots \prec r_{k-m} \prec r_{k+1} \prec$ $\ldots \prec r_{l}$. These entries are distinguished by placing a bar over them.

$\mathcal{T}_{m}=\mathcal{T}_{m}(\theta, \mu, \nu)$ is defined to be the set of all such triples $(k, R, T)$ as defined above. We define two weights on such a triple $(k, R, T)$, a positive and a negative weight, by

$$
\begin{gathered}
w^{+}(k, R, T)=\beta^{|T|-|\theta|} F_{T}\left(\left.\rho^{(l)} \underset{r_{l}}{|\cdots|}\right|_{r_{k+1}} \rho^{(k)} \underset{r_{k-m}}{\mid} \cdots \mid \rho_{r_{1}} \rho^{(0)}\right), \\
w^{-}(k, R, T)=\beta^{|T|-|\theta|} F_{T}\left(\left.\rho^{(l)}\right|_{r_{l}} \cdots\left|\rho_{r_{k+1}} \rho^{(k-m)} \underset{r_{k-m}}{\mid} \cdots\right| \rho_{r_{1}} \rho^{(0)}\right) \frac{1+\beta\left(a_{\rho^{(k)}}\right)_{r_{k+1}}}{1+\beta\left(a_{\left.\rho^{(k-m)}\right)}\right)_{r_{k+1}}} .
\end{gathered}
$$

The extra factor in the definition of $w^{-}$is to 'correct' the contribution to the product provided by the barred $r_{k+1}$. 
Let

$$
S_{m}=\sum_{(k, R, T) \in \mathcal{T}_{m}} w^{+}(k, R, T) \Pi\left(\rho^{(k-m)}\right)-\sum_{(k, R, T) \in \mathcal{T}_{m}} w^{-}(k, R, T) \Pi\left(\rho^{(k-m)}\right) .
$$

Say that $(k, R, T)$ is a positive $\varepsilon$-triple if $\rho^{(k)} / \rho^{(k-m-1)}$ contains the shape $甘$. Say that $(k, R, T)$ is a negative $\varepsilon$-triple if $\rho^{(k+1)} / \rho^{(k-m)}$ contains the shape $由$. Let $\mathcal{T}_{m}^{+}=$ $\left\{(k, R, T) \in \mathcal{T}_{m} \mid(k, R, T)\right.$ is a positive $\epsilon$-triple $\}$ and similarly $T_{m}^{-}=\left\{(k, R, T) \in \mathcal{T}_{m} \mid\right.$ $(k, R, T)$ is a negative $\epsilon$-triple $\}$. Define

$$
\varepsilon_{m}=\sum_{(k, R, T) \in \mathcal{T}_{m}^{+}} w^{+}(k, R, T) \Pi\left(\rho^{(k-m)}\right)-\sum_{(k, R, T) \in \mathcal{T}_{m}^{-}} w^{-}(k, R, T) \Pi\left(\rho^{(k-m)}\right) .
$$

$S_{0}=0$, as $w^{+}$and $w^{-}$are identical functions when $m=0 . S_{l+1}=0$, as there are no sequences with $m=l+1$. Similarly $\varepsilon_{0}=\varepsilon_{m+1}=0$. Now if we temporarily assume Proposition 6.2 below, we can obtain the equation

$$
\sum_{\mu \rightrightarrows \lambda} \beta^{|\lambda / \mu|} g_{\theta \lambda}^{\nu} \Pi(\mu)=\sum_{\eta \rightrightarrows \nu} \beta^{|\nu / \eta|} g_{\theta \mu}^{\eta} \Pi(\eta)
$$

which is equivalent to the recurrence, so we are done.

So the proof of Theorem 6.1 follows immediately from the proof of the following proposition.

\section{Proposition 6.2.}

$$
S_{m}-\varepsilon_{m}=\beta\left(S_{m+1}-\varepsilon_{m+1}\right)+\sum_{\substack{\mu \rightrightarrows \lambda \\|\lambda / \mu|=m}} g_{\theta \lambda}^{\nu} \Pi(\mu)-\sum_{\substack{\eta \rightrightarrows \nu \\|\nu / \eta|=m}} g_{\theta \mu}^{\eta} \Pi(\eta)
$$

Proof. The positive terms in $S_{m}$ with $k=m$ and the negative terms in $S_{m}$ with $k=l$ give exactly

$$
\sum_{\substack{\mu \rightrightarrows \lambda \\|\lambda / \mu|=m}} g_{\theta \lambda}^{\nu} \Pi(\mu)-\sum_{\substack{\eta \rightrightarrows \nu \\|\nu / \eta|=m}} g_{\theta \mu}^{\eta} \Pi(\eta)
$$

So now we consider positive terms in $S_{m}$ with $k>m$, and negative terms with $k<l$. For positive terms, we consider $\Theta=\rho^{(k)} / \rho^{(k-m-1)}$ while for negative terms we consider $\Theta=\rho^{(k+1)} / \rho^{(k-m)}$. We have two separate cases to consider, according to the shape of $\Theta$.

Case 1: $\Theta$ contains two boxes in the same row.

Consider a positive term $w^{+}(k, R, T)$ covered by this case. Define $\left(k^{\prime}, R^{\prime}, T^{\prime}\right)$ as follows: Set $k^{\prime}=k-1$. Construct $R^{\prime}$ from $R$ by replacing the subsequence $\rho^{(k-m-1)} \stackrel{r_{k-m}}{\longrightarrow} \rho^{(k-m)} \rightrightarrows$ $\rho^{(k)}$ by $\rho^{(k-m-1)} \rightrightarrows \rho^{\prime} \stackrel{r_{k-m}}{\longrightarrow} \rho^{(k)}$ (there exists a unique such partition $\rho^{\prime}$ ). Set $T^{\prime}=T$.

Then $w^{+}(k, R, T) \Pi\left(\rho^{(k-m)}\right)=w^{-}\left(k^{\prime}, R^{\prime}, T^{\prime}\right) \Pi\left(\rho^{\left(k^{\prime}-m\right)}\right)$. For the only factor differing in $w^{+}(k, R, T)$ and $w^{-}\left(k^{\prime}, R^{\prime}, T^{\prime}\right)$ is that due to the barred $r_{k-m}$. In $w^{+}(k, R, T)$, this entry contributes the factor $\left(1+\beta\left(a_{\rho^{(k-m-1)}}\right)_{r_{k-m}}\right)\left(1+\beta b_{r_{k-m}+c(\alpha)}\right)$ while in $w^{-}\left(k^{\prime}, R^{\prime}, T^{\prime}\right)$, this 
entry contributes $\left(1+\beta\left(a_{\rho^{\prime}}\right)_{r_{k-m}}\right)\left(1+\beta b_{r_{k-m}+c(\alpha)}\right)$, precisely countering the difference in the factors $\Pi\left(\rho^{(k-m)}\right)$ and $\Pi\left(\rho^{\left(k^{\prime}-m\right)}\right)$.

This map $(k, R, T) \mapsto\left(k^{\prime}, R^{\prime}, T^{\prime}\right)$ has a similar inverse, hence is bijective, so we have shown that all terms of $S_{m}$ which are covered by this case cancel each other to give no net contribution.

Case 2: All boxes of $\Theta$ are in different rows and columns.

Given such a positive triple $\left(k_{0}, R_{0}, T_{0}\right)$, consider all such triples $(k, R, T)$ with $k=k_{0}$, $T=T_{0}$ and $R=R_{0}$ except for $\rho^{(k-m)}$ (so there are $m+1$ such triples). Also consider all negative triples $\left(k^{\prime}, R^{\prime}, T^{\prime}\right)$ with $k^{\prime}=k-1, T^{\prime}=T_{0}$ and $R=R_{0}$ except that the subsequence $\rho^{(k-m-1)} \rightarrow \rho^{(k-m)} \rightrightarrows \rho^{(k)}$ is replaced by $\rho^{(k-m-1)} \rightrightarrows \rho^{\prime} \rightarrow \rho^{(k)}$ for one of the $m+1$ possibilities for $\rho^{\prime}$.

Let the row numbers of $\rho^{(k)} / \rho^{(k-m-1)}$ be $s_{1}, s_{2}, \ldots, s_{m+1}$. Let $y_{i}=\left(a_{\rho^{(k)}}\right)_{i}, z_{i}=$ $\left(a_{\rho^{(k-m-1)}}\right)_{i}$.

Then these $2 m+2$ triples together contribute the following to the sum $S_{m}$ :

$$
\beta^{|T|-|\theta|} \sum_{j=1}^{m+1} F_{T}\left(\cdots\left|\rho^{(k)}\right|_{s_{j}} \rho^{(k-m-1)} \mid \cdots\right)\left(\frac{1+\beta y_{s_{j}}}{1+\beta z_{s_{j}}}\right) \Pi\left(\rho^{(k-m-1)}\right)
$$

Between $\overline{r_{k+1}}$ and $\overline{r_{k-m-1}}$, suppose the $s_{j}$ 's (all possible $j$ ) occur in order $t_{1} \prec t_{2} \prec$ $\cdots \prec t_{p}$ and suppose $t_{i}$ lies in cell $\alpha_{i}$.

We only need to consider the entries $t_{1}, \ldots, t_{p}$ in $T$, for all other entries, along with $\Pi\left(\rho^{(k-m-1)}\right)$ contribute a common factor. After taking out that very common factor, and noticing that the relevant barred $s_{j}$ contributes a factor $\left(1+\beta z_{s_{j}}\right)\left(1+\beta b_{s_{j}+c\left(\alpha_{j}\right)}\right)$, we get

$$
\begin{aligned}
& \sum_{i=1}^{p} \beta\left(y_{t_{i}}-z_{t_{i}}\right)\left(1+\beta b_{t_{i}+c\left(\alpha_{i}\right)}\right) \prod_{j=1}^{i-1} y_{t_{j}} \oplus b_{t_{j}+c\left(\alpha_{j}\right)} \prod_{j=i+1}^{p} z_{t_{j}} \oplus b_{t_{j}+c\left(\alpha_{j}\right)} \\
= & \beta \sum_{i=1}^{p}\left(y_{t_{i}} \oplus b_{t_{i}+c\left(\alpha_{i}\right)}-z_{t_{i}} \oplus b_{t_{i}+c\left(\alpha_{i}\right)}\right) \prod_{j=1}^{i-1} y_{t_{j}} \oplus b_{t_{j}+c\left(\alpha_{j}\right)} \prod_{j=i+1}^{p} z_{t_{j}} \oplus b_{t_{j}+c\left(\alpha_{j}\right)} .
\end{aligned}
$$

This is a telescoping sum and equals

$$
\beta\left(\prod_{j=1}^{p} y_{t_{j}} \oplus b_{t_{j}+c\left(\alpha_{j}\right)}-\prod_{j=1}^{p} z_{t_{j}} \oplus b_{t_{j}+c\left(\alpha_{j}\right)}\right) .
$$

Now if we replace the common factor, we obtain

$$
\beta\left(w^{+}\left(k, R^{*}, T\right)-w^{-}\left(k, R^{*}, T\right)\right) \Pi\left(\rho^{(k-(m+1))}\right)
$$

where $R^{*}$ is the sequence obtained by replacing $\rho^{(k-m-1)} \rightarrow \rho^{(k-m)} \rightrightarrows \rho^{(k)}$ by $\rho^{(k-(m+1))} \rightrightarrows$ $\rho^{(k)}$.

Hence, when considering the contribution of all terms of $S_{m}$ covered by this case, they add up to give exactly $\beta S_{m+1}$. 
Case 3: $\Theta$ contains two boxes in the same column, but does not contain two boxes in the same row.

Let $i$ and $i+1$ be the row numbers of the two boxes of $\Theta$ which are in the same column. We will underline the marked $i$ and $i+1$ in $T$ which come from $\Theta$ for increased clarity.

Tableaux containing the following cannot occur as they cannot arise from a sequence of partitions.

\section{\begin{tabular}{|l|l|l|l|l|l|}
\hline$\underline{i}$ & $\cdots$ & $\bar{i}$ \\
\hline$i+1$ & $\cdots$ & $\overline{i+1}$ \\
\hline
\end{tabular}}

If $\underline{i}$ appears to the left of $\bar{i}$ in the same row, then by examining $R$, there would need to be a marked $i+1$ between these two entries in reverse column order. But since $\theta$ does not contain two boxes in the same column, this cannot happen for the tableau to be semistandard. The case of $\underline{i+1}$ to the right of $\overline{i+1}$ in the same row proceeds similarly.

Wherever possible, we match up our tableaux as follows:

Given an $\underline{i+1}$ in a negative term, denote by $L$ the box with this entry and all consecutive boxes to its left which contain an $i+1$. Consider the two tableaux $T_{1}$ and $T_{2}$, the first with an unbarred $i$ in the first box of $L$ and the second without this $i$. We match these up with $T_{3}$ and $T_{4}$ which are obtained by changing all $i$ 's and $i+1$ 's in $L$ such that $\bar{i}$ is in the leftmost box of $L$, an unbarred $i$ is in each other box of $L$ and $T_{3}$ contains an unbarred $i+1$ in the rightmost box of $L$ while $T_{4}$ does not. For example $(\mathrm{i}=2)$ :

$$
\begin{aligned}
& T_{1}+T_{2} \longleftrightarrow T_{3} \quad \longleftrightarrow \quad T_{4} \\
& \begin{array}{|l|l|l|}
\hline 23 & 3 & \underline{\overline{3}} \\
\hline
\end{array}+\begin{array}{|l|l|l|}
\hline 3 & 3 & \underline{\overline{3}} \\
\hline
\end{array} \longleftrightarrow \begin{array}{|l|l|l|}
\hline \underline{\overline{2}} & 2 & 23 \\
\hline
\end{array}
\end{aligned}
$$

Now we shall show that under this identification, the corresponding terms of $S_{m}$ cancel, that is

$$
w^{-}\left(k-1, R^{\prime}, T_{1}\right)+w^{-}\left(k-1, R^{\prime}, T_{2}\right)=w^{+}\left(k, R, T_{3}\right)+w^{+}\left(k, R, T_{4}\right)
$$

where $R^{\prime}$ is the sequence obtained from $R$ by replacing $\rho^{(k-m-1)} \rightarrow \rho^{(k-m)} \rightrightarrows \rho^{(k)}$ with $\rho^{(k-m-1)} \rightrightarrows \rho^{\prime} \rightarrow \rho^{(k)}$.

This is because the two sides of the equation have common factors from their common entries, as well as from the unmarked $i$ 's in the positive terms and the unmarked $i+1$ 's in the negative terms. Apart from these common factors, the positive terms have, upon combination, the extra factors $(1+\beta v)\left(1+\beta b_{i+c\left(\alpha_{l}\right)}\right)$ from $\underline{\underline{i}},(1+\beta w)\left(1+\beta b_{i+1+c\left(\alpha_{r}\right)}\right)$ from $i+1$ and $1+\beta u$ from $\Pi\left(\rho^{(k-m)}\right)$, while the negative terms have the extra factors $(1+\beta w)(1+$ $\left.\beta b_{i+1+c\left(\alpha_{r}\right)}\right)$ from $\overline{i+1},(1+\beta u)\left(1+\beta b_{i+c\left(\alpha_{l}\right)}\right)$ from $i$ and $1+\beta v$ from $\Pi\left(\rho^{(k-m-1)}\right)$. Here $u=\left(a_{\rho^{(k)}}\right)_{i}, v=\left(a_{\rho^{(k)}}\right)_{i+1}, w=\left(a_{\rho^{(k-m)}}\right)_{i+1}$ and $\alpha_{l}$ and $\alpha_{r}$ are respectively the leftmost and rightmost boxes of $L$.

Taking into account those tableaux which we have already shown to give zero contribution to the sum, we find that the only remaining tableaux $T$ for which the above identification of positive and negative terms cannot be made, is where a barred $i$ is in the leftmost cell of $L$ ( $L$ as defined above) for a negative term and vice versa for a positive 
term. We shall focus our attention on the positive terms for which this happens, as the negative case proceeds similarly.

Then the following must occur as a subsequence of $R$ :

$$
\rho^{(k-m-2)} \stackrel{i+1}{\longrightarrow} \rho^{(k-m-1)} \stackrel{i}{\longrightarrow} \rho^{(k-m)} \rightrightarrows \rho^{(k)}
$$

By replacing this with $\rho^{(k-m-2)} \rightrightarrows \rho^{\prime} \stackrel{i+1}{\longrightarrow} \rho^{(k)}$, we form another sequence $R^{\prime}$.

We map $T$ with $i$ barred in the leftmost box of $L$ to $T^{\prime}$ and $T^{\prime \prime}$ with the barred $i$ removed in the first case and unbarred in the second case. All boxes of $L$ in $T^{\prime}$ and $T^{\prime \prime}$ contain an $i+1$ with the rightmost of these barred. For example:

$$
\begin{array}{|l|l|l|l|l|l|}
\hline \underline{\overline{2}} & 2 & 2 \overline{3} & \mapsto 3 & 3 & \overline{3} \\
\hline
\end{array}
$$

Lemma 6.3.

$$
w^{+}(k, R, T) \Pi\left(\rho^{(k-m)}\right)=\beta\left(w^{-}\left(k-1, R^{\prime}, T^{\prime}\right)+w^{-}\left(k-1, R^{\prime}, T^{\prime \prime}\right)\right) \Pi\left(\rho^{(k-m-2)}\right) .
$$

Proof. Define $u, v, w, x$ by the following, where the pronumeral in column $\rho$ and row $j$ represents $\left(a_{\rho}\right)_{j}$.

\begin{tabular}{c|c|c|c|c} 
& $\rho^{(k-m-2)}$ & $\rho^{(k-m-1)}, \rho^{\prime}$ & $\rho^{(k-m)}$ & $\rho^{(k)}$ \\
\hline$i$ & $v$ & $v$ & $u$ & $u$ \\
$i+1$ & $x$ & $w$ & $w$ & $v$
\end{tabular}

Each side of (6.3) has common factors due to common entries and from the unbarred $i$ 's and $i+1$ 's in $L$ in the positive and negative terms respectively. Aside from these common factors, the left hand side of $(6.3)$ has factors $(1+\beta v)\left(1+\beta b_{i+c\left(\alpha_{l}\right)}\right)$ from $\underline{\bar{i}}$, $(1+\beta x)\left(1+\beta b_{i+1+c\left(\alpha_{r}\right)}\right)$ from $\overline{i+1}$ and $(1+\beta u)(1+\beta w)$ from $\Pi\left(\rho^{(k-m)}\right)$, while the right hand side has factors $(1+\beta w)\left(1+\beta b_{i+1+c\left(\alpha_{r}\right)}\right)$ from $\overline{i+1},(1+\beta u)\left(1+\beta b_{i+c\left(\alpha_{l}\right)}\right)$ from $i$ and $(1+\beta v)(1+\beta x)$ from $\Pi\left(\rho^{(k-m-2)}\right)$. Again $\alpha_{l}$ and $\alpha_{r}$ denote the leftmost and rightmost cells of $L$. The extra factor of $\beta$ is due to there being one more entry in $T$ than in $T^{\prime}$.

We note that the inverse to this map can always be created, for given a tableau related to a sequence of the form $R^{\prime}$, analysis of the sequence of partitions shows that a barred $i+1$ can never be to the left of the relevant barred $i+1$, ensuring an uninterrupted string of unbarred $i+1$ 's to the left of the relevant barred $i+1$ which enables the inverse to be easily constructed. Thus positive terms covered by Case 3 in $S_{m}$ give exactly $\beta$ times the negative terms in $\varepsilon_{m+1}$. Similarly, we see that the negative terms covered by Case 3 in $S_{m}$ give $\beta$ times the positive terms in $\varepsilon_{m+1}$.

Case 4: The only remaining triples are $\varepsilon$-triples, so their contribution to $S_{m}$ is exactly $\varepsilon_{m}$.

Hence we have proven (6.3), as desired. 


\subsection{Partial solution in the general case}

We now consider the case where $b=0$, with a view to turning our attention to the ordinary Grothendieck polynomials. We return to the situation where $\theta$ is an arbitrary skew diagram. In this case, we provide a partial solution to the recurrence relation. We shall carry over notation used in the case of arbitrary $b$, just noting that the variables $b_{i}$ are all to be set equal to zero.

We shall also set the following variables $a_{i}$ equal to zero: If there exists a sequence $R$ of the form (6.1), and a $k$ for which $\rho^{(k+1)} / \rho^{(k-1)}$ consists of two boxes in the same column, then set $\left(a_{\rho^{(k-1)}}\right)_{r_{k}}=0$. Let us pause and note that this is equivalent to $\left(a_{\rho^{(k+1)}}\right)_{r_{k+1}}=0$.

Theorem 6.4. If the appropriate variables are all set to zero as described above, then we have

$$
g_{\theta \mu}^{\nu}(a, 0)=\sum_{(R, T)} \beta^{|T|-|\theta|} w(T) .
$$

where again, the sum is over all $\theta$-tableaux $T$ which are related to a sequence $R$ of the form (6.1).

Proof. As in the proof of Theorem 6.1, this proposed solution satisfies the boundary conditions of the recurrence. So now we suppose that $\mu \subsetneq \nu$. Despite setting some of the variables $a_{i}$ equal to zero, we still have $\Pi(\nu) \neq \Pi(\mu)$, so we are able to calculate the coefficients using the recurrence (5.4). So it now suffices to show that our proposed solution satisfies the recurrence. So we suppose that (6.5) holds and use this to show that (5.4) holds.

Again as in the proof of Theorem 6.1, the proof reduces to the proof of the following proposition.

\section{Proposition 6.5.}

$$
S_{m}-\varepsilon_{m}=\beta\left(S_{m+1}-\varepsilon_{m+1}\right)+\sum_{\substack{\mu \rightrightarrows \lambda \\|\lambda / \mu|=m}} g_{\theta \lambda}^{\nu} \Pi(\mu)-\sum_{\substack{\eta \rightrightarrows \nu \\|\nu / \eta|=m}} g_{\theta \mu}^{\eta} \Pi(\eta)
$$

Proof. As per the proof of Proposition 6.2, in $S_{m}$, the positive terms with $k=m$ and the negative terms with $k=l$ give exactly

$$
\sum_{\substack{\mu \rightrightarrows \lambda \\|\lambda / \mu|=m}} g_{\theta \lambda}^{\nu} \Pi(\mu)-\sum_{\substack{\eta \rightrightarrows \nu \\|\nu / \eta|=m}} g_{\theta \mu}^{\eta} \Pi(\eta)
$$

For the remaining terms in $S_{m}$, we split them up into three cases according to the shape of $\Theta$.

Case 1: $\Theta$ contains two boxes in the same row, and does not contain two boxes in the same column.

This case is the same as in the previous solution, all such $\Theta$ combined contribute zero to the sum. 
Case 2: $\Theta$ contains all boxes in different rows and columns.

Again this case is the same as in the previous solution, contributing $\beta S_{m+1}$ to the sum.

Case 3: $\Theta$ contains two boxes in the same column, but does not contain two boxes in the same row.

Let $i$ and $i+1$ be the row numbers of the two boxes of $\Theta$ which are in the same column. We will underline the marked $i$ and $i+1$ in $T$ which come from $\Theta$ for increased clarity.

Tableaux containing the following have zero weight, due to setting variables equal to zero, so their contribution can be neglected:

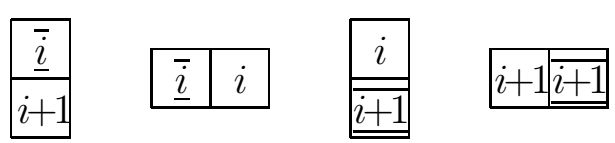

This is because, in the first case, the $i+1$ contributes $\left(a_{\rho^{(k)}}\right)_{i+1}=0$ to the product $w^{+}(k, R, T)$, while in the second case, the $i$ contributes $\left(a_{\lambda}\right)_{i}$ for some partition $\lambda$ occuring in the sequence $R$. Since there are no occurrences of $i$ in $T$ between this $i$ and the relevant marked $i$ in reverse column order, there cannot be any occurrences of $\stackrel{r_{i}}{\longrightarrow}$ in $R$ between $\lambda$ and $\rho^{(k-m-1)}$, so $\left(a_{\lambda}\right)_{i}=\left(a_{\rho^{(k-m-1)}}\right)_{i}=0$. Hence this tableau has $w^{+}(k, R, T)$ and can safely be ignored. For the final two cases, a similar argument shows that they give tableaux for which $w^{-}(k, R, T)=0$.

Tableaux containing the following either cannot occur as they cannot arise from a sequence of partitions or contribute zero to the sum as in the above, so can also be ignored.

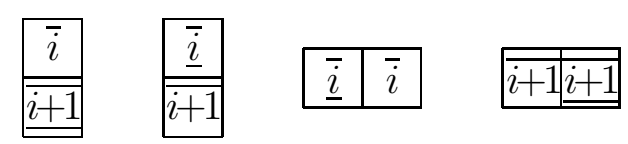

This is because $\underline{i}$ above $\overline{i+1}$ or $\bar{i}$ above $\overline{i+1}$ cannot arise from a sequence of partitions $R$. If $\underline{i}$ to the immediate left of $\bar{i}$, then by examining $R$, there would need to be a marked $i+1$ between these two entries in reverse column order, which can only lie directly below $\bar{i}$. But then to be semistandard, the entry directly below $\underline{i}$ must be an $i+1$. We have already shown that this entry cannot be marked. So it is unmarked, in which case we have already shown that the tableau gives zero contribution so can be ignored. The case of $\overline{i+1}$ to the immediate right of $\overline{i+1}$ proceeds similarly.

Wherever possible, we match up our tableaux as such:

$$
\underline{\underline{i}}+\underline{\underline{\bar{i}}, i+1} \leftrightarrow \underline{\overline{i+1}}+\overline{i, \overline{i+1}}
$$

where all other elements of $T$ are unchanged. Then making this pairing, the corresponding terms in $S_{m}$ cancel as it is a special case of (6.4). Taking into account those tableaux which we have already shown to give zero contribution to the sum, we find that the only remaining tableaux $T$ for which the above identification of positive and negative terms cannot be made, is where $i$ and $i+1$, both barred and one underlined occur in the same 
cell of $T$. We shall focus our attention on the positive terms for which this happens, as the negative case proceeds similarly.

Then the following must occur as a subsequence of $R$ :

$$
\rho^{(k-m-2)} \stackrel{i+1}{\longrightarrow} \rho^{(k-m-1)} \stackrel{i}{\longrightarrow} \rho^{(k-m)} \rightrightarrows \rho^{(k)} .
$$

By replacing this with $\rho^{(k-m-2)} \rightrightarrows \rho^{\prime} \stackrel{i+1}{\longrightarrow} \rho^{(k)}$, we form another sequence $R^{\prime}$.

We map $T$ with $i$ and $i+1$ barred in the same box to $T^{\prime}$ and $T^{\prime \prime}$ with the barred $i$ removed in the first case and unbarred in the second case.

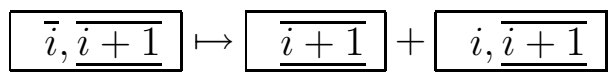

Again we have Lemma 6.3,

$$
w^{+}(k, R, T) \Pi\left(\rho^{(k-m)}\right)=\beta\left(w^{-}\left(k-1, R^{\prime}, T^{\prime}\right)+w^{-}\left(k-1, R^{\prime}, T^{\prime \prime}\right)\right) \Pi\left(\rho^{(k-m-2)}\right) .
$$

with the same proof.

Now we look at when the inverse to this map can be constructed:

$$
\underline{\overline{i+1}}+\underline{i, \underline{i+1}} \mapsto \overline{\bar{i}, \underline{i+1}}
$$

Here we will underline the relevant $\overline{i+1}$ for clarity.

We consider the cases when this map cannot be made. They are

(i) Unbarred $i+1$ to the immediate left of $\overline{i+1}$ : The unbarred $i+1$ contributes $\left(a_{\rho^{(k)}}\right)_{i+1}=0$ to the product, so these tableaux can be ignored.

(ii). Unbarred $i$ above $\underline{\overline{i+1}}$ : The unbarred $i$ contributes $\left(a_{\left.\rho^{(k-m-2)}\right)}\right)_{i}=0$ to the product, so again we have zero contribution so these tableaux can be ignored. ignored.

(iii). $\overline{i+1}$ to the immediate left of $\overline{i+1}$ : In order to have a sequence of partitions, there must be a barred $i$ between these two barred $i+1$ 's, so must lie directly above the non-underlined one. Thus, above $\overline{i+1}$ must either lie an unbarred $i$, giving case (ii), or a barred $i$, giving the final case which we now deal with.

(iv). $\bar{i}$ immediately above $\overline{i+1}$ : We note that in this case the sequence must contain the subsequence $\rho^{(k-m-3)} \stackrel{i}{\longrightarrow} \rho^{(k-m-2)} \rightrightarrows \rho^{\prime} \stackrel{i+1}{\longrightarrow} \rho^{(k)}$.

Thus positive terms covered by Case 3 in $S_{m}$ give $\beta$ times the negative terms in $\varepsilon_{m+1}$, with the exception of those terms which are covered by case (iv) above.

Similarly, we see that the negative terms covered by Case 3 in $S_{m}$ give $\beta$ times the positive terms in $\varepsilon_{m+1}$ with the same exception of those terms covered in case (iv).

Using our observation of the structure of case (iv) terms, we note that if $(k, R, T)$ is such a positive $\varepsilon$-triple, then it is also a negative $\varepsilon$-triple. Furthermore for such terms $w^{+}(k, R, T)=w^{-}(k, R, T)$, so it is seen that such terms cancel themselves out, giving no net contribution, and hence the contribution to $S_{m}$ by all triples covered by this case is exactly $-\beta \varepsilon_{m+1}$.

Case 4: The only remaining triples are $\varepsilon$-triples, so their contribution to $S_{m}$ is exactly $\varepsilon_{m}$.

Hence we have proven (6.6), as desired. 
Remark 6.6. It is necessary for us to set some variables equal to zero in (6.5), as otherwise, the formula does not hold true. For example, if $n=2, \theta=\nu=\left(1^{2}\right)$ and $\mu=\phi$, then using the recurrence, we calculate $g_{\theta \mu}^{\nu}=\frac{1+\beta b_{1}}{1+\beta a_{1}}$ whereas $\sum_{(R, T)} \beta^{|T|-|\theta|} w(T)=\frac{\left(1+\beta b_{1}\right)^{2}}{\left(1+\beta a_{1}\right)\left(1+\beta a_{2}\right)}$.

\subsection{Specialisation to ordinary Grothendieck polynomials}

Specialisation to $a=0$ in (5.3) gives $g_{\theta \mu}^{\nu}(0,0)=c_{\theta \mu}^{\nu}$, where $c_{\theta \mu}^{\nu}$ is as defined in (3.3). Under this specialisation, a pair $(R, T)$ will contribute $\beta^{|T|-|\theta|}$ to the sum in (6.5) if it consists entirely of barred entries, and 0 otherwise. For such tableaux, we must thus have $|T|=|\nu|-|\mu|$. Hence we have the following.

Theorem 6.7. $c_{\theta \mu}^{\nu}$ is equal to $\beta^{|\nu|-|\mu|-|\theta|}$ times the number of semistandard set-valued $\theta$ tableaux with entries $r_{1} \prec r_{2} \prec \ldots \prec r_{l}$ for which there is a related sequence of partitions

$$
\mu=\rho^{(0)} \stackrel{r_{1}}{\longrightarrow} \rho^{(1)} \stackrel{r_{2}}{\longrightarrow} \cdots \stackrel{r_{l}}{\longrightarrow} \rho^{(l)}=\nu .
$$

We can show directly that this specialises to Buch's results as quoted in Theorems 3.10 and 3.11 .

In the case $\theta=\lambda$, a partition, in order to directly see that our result is equivalent to Theorem 3.10, we note that if $T$ is a $\mu$-tableau and $c(T)$ is to be a lattice word, then the entries of $T$ are fixed, namely in that the $i$-th row must contain only $i$ 's. Call this particular tableau $T_{\mu}$. Now for $c\left(T^{\prime}\right)$ to be a lattice word for some $\lambda * \mu$-tableau $T^{\prime}$, we must have $T^{\prime}=T * T_{\mu}$ for some $\lambda$-tableau $T$. Now we have a simple bijection between the two formulations in this case, namely that which is given by $T \mapsto T * T_{\mu}$. The condition of $c\left(T * T_{\mu}\right)$ being a lattice word is equivalent to the sequence

$$
\mu=\rho^{(0)} \stackrel{r_{1}}{\longrightarrow} \rho^{(1)} \stackrel{r_{2}}{\longrightarrow} \cdots \stackrel{r_{l}}{\longrightarrow} \rho^{(l)}=\nu .
$$

consisting entirely of partitions.

For the case $\mu=\phi$, where we are expanding a skew Grothendieck polynomial in the basis of ordinary Grothendieck polynomials, Theorem 3.11 is easily seen to be consistent with our formulation since $r_{1}, r_{2}, \ldots, r_{m}$ is a lattice word if and only if

$$
\phi=\rho^{(0)} \stackrel{r_{1}}{\longrightarrow} \rho^{(1)} \stackrel{r_{2}}{\longrightarrow} \cdots \stackrel{r_{m}}{\longrightarrow} \rho^{(m)}=\lambda
$$

is a sequence of partitions where $\lambda$ is the content of $r_{1}, r_{2}, \ldots, r_{m}$.

\section{Grothendieck Polynomials via Isobaric Divided Differences}

The remainder of this paper will have a distinctly different flavour to it, as we move away from calculating the Littlewood-Richardson coefficients and instead devote the remainder of our energies to exhibiting a relationship between the factorial Grothendieck polynomials studied here, and the double Grothendieck polynomials, as studied elsewhere. For the most part of this section, we follow the exposition of Fomin and Kirillov [5], supplying some proofs which are missing in their extended abstract. 


\subsection{The symmetric group}

It is well known that the symmetric group $S_{n+1}$ is generated by the $n$ simple reflections $s_{i}=(i, i+1), i=1,2, \ldots, n$ subject to the relations

$$
\begin{array}{rlr}
s_{i} s_{j} & =s_{j} s_{i} \quad \text { if }|i-j| \geq 2, \\
s_{i} s_{i+1} s_{i} & =s_{i+1} s_{i} s_{i+1}, & \\
s_{i}^{2} & =1 .
\end{array}
$$

For an element $w \in S_{n+1}$, let $\ell(w)$ denote the minimal number $l$ for which $w$ can be written as a product of $l$ simple reflections $w=s_{i_{1}} s_{i_{2}} \ldots s_{i_{l}}$. Then $\ell(w)=\#\{i<j \mid w(i)>w(j)\}$. The longest word in $S_{n+1}$ is $w_{0}=(n+1, n, \ldots, 1)$ and satisfies $\ell\left(w_{0}\right)=\frac{n(n+1)}{2}$.

\subsection{Isobaric divided difference operators}

Let $R$ be a commutative ring with identity. We shall also assume that $R$ contains various indeterminates used later, namely $\beta, a_{1}, a_{2}, \ldots$ Let $f$ be a polynomial in the variables $x_{1}, x_{2}, \ldots, x_{n+1}$ over $R$. For $i=1,2, \ldots, n$, define the isobaric divided difference operator $\pi_{i}$ by

$$
\pi_{i} f=\frac{\left(1+\beta x_{i+1}\right) f\left(\ldots, x_{i}, x_{i+1}, \ldots\right)-\left(1+\beta x_{i}\right) f\left(\ldots, x_{i+1}, x_{i}, \ldots\right)}{x_{i}-x_{i+1}}
$$

Then these isobaric divided difference operators are easily verified to satisfy the following relations:

$$
\begin{array}{rlr}
\pi_{i} \pi_{j} & =\pi_{j} \pi_{i} \quad \text { if }|i-j| \geq 2, \\
\pi_{i} \pi_{i+1} \pi_{i} & =\pi_{i+1} \pi_{i} \pi_{i+1}, \\
\pi_{i}^{2} & =-\beta \pi_{i} .
\end{array}
$$

For each permutation $w \in S_{n+1}$ we now define the Grothendieck polynomial $\mathcal{G}_{w}$ in $x_{1}, x_{2} \ldots, x_{n+1}, y_{1}, y_{2}, \ldots y_{n+1}$. (We shall see later that these are actually polynomials only in the variables $x_{1}, \ldots, x_{n}, y_{1}, \ldots, y_{n}$ but this will not be immediately apparent.) If $w=w_{0}$, the longest permutation, then set

$$
\mathcal{G}_{w_{0}}=\prod_{i+j \leq n+1}\left(x_{i} \oplus y_{j}\right)
$$

If $w \neq w_{0}$, then there exists a simple reflection $s_{i}$ such that $\ell\left(w s_{i}\right)>\ell(w)$. In such a case, we set $\mathcal{G}_{w}=\pi_{i} \mathcal{G}_{w s_{i}}$. This definition is independent of the choice of simple reflection, since the operators $\pi_{i}$ satisfy the Coxeter relations (7.1) and (7.2).

\subsection{The algebra $H_{n}$.}

Let $H_{n}$ be the $R\left[x_{1}, \ldots, x_{n}\right]$-algebra generated by $u_{1}, u_{2}, \ldots, u_{n}$ subject to

$$
\begin{array}{rlr}
u_{i} u_{j} & =u_{j} u_{i} \quad \text { if }|i-j| \geq 2, \\
u_{i} u_{i+1} u_{i} & =u_{i+1} u_{i} u_{i+1}, \\
u_{i}^{2} & =\beta u_{i} .
\end{array}
$$


Then $H_{n}$ has dimension $(n+1)$ ! and a natural basis $u_{w}$ indexed by elements of $S_{n+1}$ where $u_{w}=u_{i_{1}} \ldots u_{i_{l}}$ if $w=s_{i_{1}} \ldots s_{i_{l}}$ is a minimal representation of $w$ as a product of simple reflections. [1, Ch 4, $§ 2$, Ex 23]

For $x \in R\left[x_{1}, \ldots, x_{n}\right]$, define the following:

$$
\begin{aligned}
h_{i}(x) & =1+x u_{i} \\
A_{i}(x) & =h_{n}(x) h_{n-1}(x) \ldots h_{i}(x) \\
B_{i}(x) & =h_{i}(x) h_{i+1}(x) \ldots h_{n}(x) \\
A(x) & =A_{1}(x) \\
B(x) & =B_{1}(x) .
\end{aligned}
$$

For $x=\left(x_{1}, x_{2}, \ldots, x_{n}\right)$, we also define

$$
\begin{aligned}
\mathcal{G}(x) & =A_{1}\left(x_{1}\right) A_{2}\left(x_{2}\right) \ldots A_{n}\left(x_{n}\right), \\
\overline{\mathcal{G}}(x) & =B_{n}\left(x_{n}\right) B_{n-1}\left(x_{n-1}\right) \ldots B_{1}\left(x_{1}\right) .
\end{aligned}
$$

Now we begin proving some preliminary identities in $H_{n}$.

Lemma 7.1. [5]

$$
B(x) B(y)=B(y) B(x) .
$$

Proof. Expand $B(x) B(y)$ as a sum of $4^{n}$ terms. We identify each of these terms with a 2-colouring of a $2 \times n$ array of boxes. The two colours chosen here are crossed and uncoloured. Each colouring $U$ is identified with the term obtained by taking $x u_{i}$ from the factor $h_{i}(x)$ (respectively $y u_{i}$ from $h_{i}(y)$ ) from the $i$-th box in the first (respectively second) row if it contains a cross and 1 otherwise. Denote this term by $\xi(U)$. So for example if

$$
U=\triangle M A
$$

\begin{tabular}{|c|c|c|c|}
\hline Suppose & $U=$ & $U_{1}$ & $U_{2}$ \\
\hline Define & $U^{\prime}=$ & $U_{1}^{\prime}$ & $U_{2}^{\prime}$ \\
\hline
\end{tabular}

then $\xi(U)=x^{3} y^{2} u_{1} u_{3} u_{5} u_{3} u_{4}$.

Let $\sigma\left(x^{i} y^{j} u_{w}\right)=x^{j} y^{i} u_{w}$.

We now find a bijection $U \rightarrow U^{\prime}$ such that $\sigma(\xi(U))=\xi\left(U^{\prime}\right)$, assuming inductively, that such a bijection exists for all such $2 \times m$ arrays with $m<n$.

There are four different types of columns that can occur in $U$, which we shall unimaginatively call types I, II, III and IV as follows:

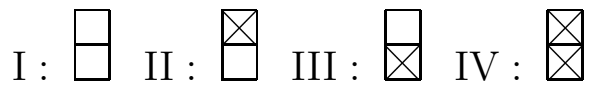

Case: $U$ contains a type I column. 
Terms from the lower half of $U_{1}$ and upper half of $U_{2}$ always commute. So as $\sigma\left(\xi\left(U_{i}\right)\right)=$ $\xi\left(U_{i}^{\prime}\right)$ for $i=1,2, \sigma(\xi(U))=\xi(U)$ as required.

So now we may assume that $U$ contains no type I columns.

Case: $U$ contains a type III column to the left of a type II column.

Of all such pairs of columns, consider a minimally separated pair. Then $U$ must contain only type IV columns between this minimally separated pair, so must be of the form

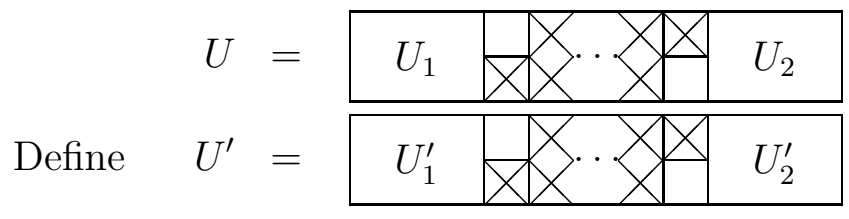

Terms from the lower half of $U_{1}$ commute with those from the upper part of $U$ to the right of $U_{1}$, and terms from the upper half of $U_{2}$ commute with those from the lower half of $U$ to the left of $U_{2}$. So, again using strong induction, from $\sigma_{i}\left(\xi\left(U_{i}\right)\right)=\xi\left(U_{i}^{\prime}\right)$, we get $\sigma(\xi(U))=\xi\left(U_{i}^{\prime}\right)$ as required.

So now may also assume that such an arrangement does not exist. Thus, we are only left to consider $U$ of the following schematic type:

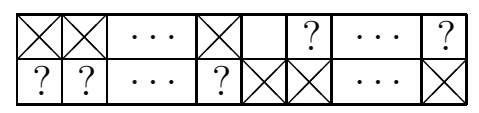

Suppose that $U$ contains $a$ type II columns and $b$ type III columns. Without loss of generality, let us assume that $a \geq b$. We can do so, since the $a<b$ case proceeds similarly, or alternatively and equivalently can be defined as the inverse of the $a>b$ case.

Let $V$ denote that part of $U$ lying strictly between the $b$-th type II column (counting from the left) and the leftmost type III column.

Draw a horizontal cutting line through the middle of $V$. Now draw a vertical cutting line one boxwidth from the right hand edge of $V$. Glue together opposite edges of $V$ to form a torus, and cut this torus along the cutting lines constructed, while preserving the directional notions of up, down, left and right to create $V^{\prime}$.

So for example if

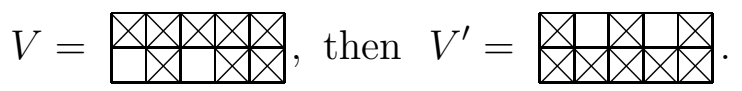

Returning to $U$, construct $U^{\prime}$ by replacing $V$ with $V^{\prime}$. It is clear that the degrees of $x$ and $y$ in $\sigma(\xi(U))$ and $\xi\left(U^{\prime}\right)$ match, while the fact that the same basis element $u_{w}$ is obtained in each case follows from the following relations:

$$
\begin{aligned}
\left(u_{i} u_{i+1} \ldots u_{k}\right) u_{j} & =u_{j+1}\left(u_{i} u_{i+1} \ldots u_{k}\right) \quad(i \leq j<k) \\
\left(u_{i} u_{i+1} \ldots u_{k}\right) u_{k} & =u_{i}\left(u_{i} u_{i+1} \ldots u_{k}\right)
\end{aligned}
$$

Thus we have our bijection $U \mapsto U^{\prime}$ such that $\sigma(\xi(U))=\xi\left(U^{\prime}\right)$ as required. Hence,

$$
B(x) B(y)=\sum_{U} \xi(U)=\sum_{U^{\prime}} \xi\left(U^{\prime}\right)=\sum_{U} \sigma(\xi(U))=B(y) B(x)
$$

and the lemma is proven. 
Corollary 7.2. [5] $A_{i}(x)$ and $A_{i}(y)$ commute, as do $A(x)$ and $A(y)$.

The following lemma is easily proven by expanding out and applying the defining relations (7.4) to (7.6). These three simple equations are used extensively in the following work.

Lemma 7.3. [5]

$$
\begin{aligned}
h_{i}(x) h_{j}(y) & =h_{j}(y) h_{i}(x) \quad \text { if }|i-j| \geq 2 \\
h_{i}(x) h_{i+1}(x \oplus y) h_{i}(y) & =h_{i+1}(y) h_{i}(x \oplus y) h_{i+1}(x) \\
h_{i}(x) h_{i}(y) & =h_{i}(x \oplus y)
\end{aligned}
$$

Lemma 7.4. $[4,5]$

$$
\left(\pi_{i}+\beta\right) \mathcal{G}(x)=\mathcal{G}(x) u_{i}
$$

(Here, $\pi_{i}$ is acting only on the elements of our coefficient ring $R\left[x_{1}, \ldots, x_{n}\right]$, so we could say that $\pi_{i}$ acts trivially on $u_{j}$ for all $j$.)

Proof. Write

$$
\mathcal{G}(x)=A_{1}\left(x_{1}\right) \ldots A_{i}\left(x_{i}\right) A_{i}\left(x_{i+1}\right) h_{i}\left(\ominus x_{i+1}\right) A_{i+2}\left(x_{i+2}\right) \ldots A_{n}\left(x_{n}\right) .
$$

A routine calculation shows that $\left(\pi_{i}+\beta\right) h_{i}\left(\ominus x_{i+1}\right)=h_{i}\left(\ominus x_{i+1}\right) u_{i}$. By Corollary 7.2, $A_{i}\left(x_{i}\right)$ and $A_{i}\left(x_{i+1}\right)$ commute so $A_{1}\left(x_{1}\right) \ldots A_{i}\left(x_{i}\right) A_{i}\left(x_{i+1}\right)$ is symmetric in $x_{i}$ and $x_{i+1}$. Since $\pi_{i}(f g)=f \pi_{i} g$ whenever $f$ is symmetric in $x_{i}$ and $x_{i+1}$, we have

$$
\begin{aligned}
& \left(\pi_{i}+\beta\right) \mathcal{G}(x) \\
& =A_{1}\left(x_{1}\right) \ldots A_{i}\left(x_{i}\right) A_{i}\left(x_{i+1}\right)\left(\pi_{i}+\beta\right) h_{i}\left(\ominus x_{i+1}\right) A_{i+2}\left(x_{i+2}\right) \ldots A_{n}\left(x_{n}\right) \\
& =A_{1}\left(x_{1}\right) \ldots A_{i}\left(x_{i}\right) A_{i}\left(x_{i+1}\right) h_{i}\left(\ominus x_{i+1}\right) u_{i} A_{i+2}\left(x_{i+2}\right) \ldots A_{n}\left(x_{n}\right) \\
& =\mathcal{G}(x) u_{i} .
\end{aligned}
$$

as required since $u_{i}$ and $A_{j}\left(x_{j}\right)$ commute for $j \geq i+2$.

Lemma 7.5. [5] $A_{i}(x)$ and $B_{i}(y)$ commute.

Proof. We prove this by descending induction on $i$.

$$
\begin{aligned}
A_{i}(x) B_{i}(y) & =h_{n}(x) \ldots h_{i+1}(x) h_{i}(x \oplus y) h_{i+1}(y) \ldots h_{n}(y) \\
& =h_{n}(x) \ldots h_{i+2}(x) h_{i}(y) h_{i+1}(x \oplus y) h_{i}(x) h_{i+2}(y) \ldots h_{n}(y) \\
& =h_{i}(y) h_{n}(x) \ldots h_{i+2}(x) h_{i+1}(x) h_{i+1}(y) h_{i+2}(y) \ldots h_{n}(y) \\
& =h_{i}(y) A_{i+1}(x) B_{i+1}(y) h_{i}(x) \\
& =h_{i}(y) B_{i+1}(y) A_{i+1}(x) h_{i}(x) \\
& =B_{i}(y) A_{i}(x) \quad \text { as required. }
\end{aligned}
$$


Lemma 7.6. [5]

$$
B_{n}\left(y_{n}\right) \ldots B_{i}\left(y_{i}\right) A_{i}(x)=h_{n}\left(x \oplus y_{n}\right) \ldots h_{i}\left(x \oplus y_{i}\right) B_{n}\left(y_{n-1}\right) \ldots B_{i+1}\left(y_{i}\right) .
$$

Proof. Again, we use descending induction on $i$.

$$
\begin{aligned}
\text { LHS } & =B_{n}\left(y_{n}\right) \ldots B_{i+1}\left(y_{i+1}\right) A_{i}(x) B_{i}\left(y_{i}\right) \\
& =B_{n}\left(y_{n}\right) \ldots B_{i+1}\left(y_{i+1}\right) A_{i+1}(x) h_{i}\left(x \oplus y_{i}\right) B_{i+1}\left(y_{i}\right) \\
& =h_{n}\left(x \oplus y_{n}\right) \ldots h_{i+1}\left(x \oplus y_{i+1}\right) B_{n}\left(y_{n-1}\right) \ldots B_{i+2}\left(y_{i+1}\right) h_{i}\left(x \oplus y_{i}\right) B_{i+1}\left(y_{i}\right) \\
& =\operatorname{RHS}
\end{aligned}
$$

since $h_{i}\left(x \oplus y_{i}\right)$ commutes with $B_{j}\left(y_{j-1}\right)$ for $j \geq i+2$.

Lemma 7.7. [5]

$$
\overline{\mathcal{G}}(y) \mathcal{G}(x)=\prod_{i=1}^{n} \prod_{j=n+1-i}^{1} h_{i+j-1}\left(x_{i} \oplus y_{j}\right) .
$$

Proof. We prove this result by induction on $n$. Lemma 7.6 gives

$$
\overline{\mathcal{G}}(y) \mathcal{G}(x)=h_{n}\left(x_{1} \oplus y_{n}\right) \ldots h_{1}\left(x_{1} \oplus y_{1}\right) B_{n}\left(y_{n-1}\right) \ldots B_{2}\left(y_{1}\right) A_{2}\left(x_{2}\right) \ldots A_{n}\left(x_{n}\right)
$$

which equals our desired result by applying the inductive hypothesis.

\subsection{A generating function for Grothendieck polynomials}

Theorem 7.8. [5]

$$
\overline{\mathcal{G}}(y) \mathcal{G}(x)=\sum_{w \in S_{n+1}} \mathcal{G}_{w} u_{w}
$$

Proof. Let $g_{w}$ be the coefficient of $u_{w}$ in $\overline{\mathcal{G}}(y) \mathcal{G}(x)$. We prove by decreasing induction on $\ell(w)$ that $g_{w}=\mathcal{G}_{w}$.

First we consider the case $w=w_{0}$. There are $\frac{n(n+1)}{2}=\ell\left(w_{0}\right)$ terms in the product on the right hand side of (7.10). We also have

$$
\left(u_{n} \ldots u_{1}\right)\left(u_{n} \ldots u_{2}\right) \ldots\left(u_{n} u_{n-1}\right)\left(u_{n}\right)=u_{w_{0}} .
$$

Hence,

$$
g_{w_{0}}=\prod_{i=1}^{n} \prod_{j=n-i+1}^{1} x_{i} \oplus y_{j}=\prod_{i+j \leq n+1} x_{i} \oplus y_{j}=\mathcal{G}_{w_{0}} .
$$

Now suppose $w \neq w_{0}$ and consider a simple reflection $s_{i}$ such that $\ell\left(w s_{i}\right)>\ell\left(w_{i}\right)$. Lemma 7.4 tells us that $\left(\pi_{i}+\beta\right) \overline{\mathcal{G}}(y) \mathcal{G}(x)=\overline{\mathcal{G}}(y) \mathcal{G}(x) u_{i}$. Comparing the coefficient of $u_{w s_{i}}$ in this equation then gives

$$
\pi_{i} g_{w s_{i}}+\beta g_{w s_{i}}=g_{w}+\beta g_{w s_{i}} .
$$

By our inductive assumption, $g_{w s_{i}}=\mathcal{G}_{w s_{i}}$, so $g_{w}=\pi_{i} \mathcal{G}_{w s_{i}}=\mathcal{G}_{w}$ as required and the theorem is proven. 
Combining (7.10) and (7.11) gives

$$
\prod_{i=1}^{n+m} \prod_{j=n+m-i}^{1} h_{i+j-1}\left(x_{i} \oplus y_{j}\right)=\sum_{w \in S_{n+m}} \mathcal{G}_{w} u_{w}
$$

If we now temporarily restrict ourselves to the finite set of variables $x_{1}, x_{2}, \ldots, x_{k}$, $y_{1}, y_{2}, \ldots, y_{l}$ (by setting $x_{i}=0$ if $i>k$ and $y_{j}=0$ if $j>l$ ), then for $m>\max (k, l)$, we apply the homomorphism $\psi: H_{n+m} \rightarrow H_{n}$ given by $\psi\left(h_{i}\right)=0$ if $i \leq m$ and $\psi\left(h_{i}\right)=h_{i-m}$ if $i>m$ to get

$$
B\left(y_{l}\right) B\left(y_{l-1}\right) \ldots B\left(y_{1}\right) A\left(x_{1}\right) A\left(x_{2}\right) \ldots A\left(x_{k}\right)=\sum_{w \in S_{n+1}} \mathcal{G}_{1^{m} \times w} u_{w} .
$$

Here, for $w \in S_{n+1}, 1^{m} \times w \in S_{m+n+1}$ is the permutation with $\left(1^{m} \times w\right)(i)=i$ if $i \leq m$ and $\left(1^{m} \times w\right)(i)=m+w(i-m)$ if $i>m$.

Thus the coefficient of each fixed monomial in $\mathcal{G}_{1^{m} \times w}$ eventually becomes stable as $m$ tends to infinity. So now we can make the following definition:

Definition 7.9. For a permutation $w$, the double stable Grothendieck polynomial in $x$ and $y$ is defined to be the power series

$$
G_{w}(x ; y)=\lim _{m \rightarrow \infty} \mathcal{G}_{1^{m} \times w} .
$$

Restricting ourselves again to the finite set of variables $x_{1}, \ldots x_{k}, y_{1} \ldots y_{l}$, we thus have

$$
B\left(y_{l}\right) B\left(y_{l-1}\right) \ldots B\left(y_{1}\right) A\left(x_{1}\right) A\left(x_{2}\right) \ldots A\left(x_{k}\right)=\sum_{w \in S_{n+1}} G_{w}(x ; y) u_{w} .
$$

Lemma 7.10. Let $p$ be an integer. Then

$$
\prod_{m=\infty}^{-\infty} \prod_{i=n}^{1} h_{i}\left(x_{m} \oplus y_{m+i-p}\right)=\sum_{w \in S_{n+1}} G_{w}(x ; y) u_{w}
$$

where any out of range variables are set equal to zero.

Proof. Repeated application of Lemma 7.6 shows the left hand side of (7.13) to equal

$$
B\left(y_{l}\right) B\left(y_{l-1}\right) \ldots B\left(y_{1}\right) A\left(x_{k}\right) A\left(x_{k-1}\right) \ldots A\left(x_{1}\right) .
$$

To complete the proof of the lemma, we use Corollary 7.2 which tells us that $A\left(x_{i}\right)$ and $A\left(x_{j}\right)$ commute, together with (7.12) and we are done. 


\section{Relationship between factorial and double Grothendieck Polynomials}

We shall restrict ourselves now to considering factorial Grothendieck polynomials $G_{\lambda}(x \mid a)$ for $\lambda$ a partition. By making such a restriction, rather than considering $G_{\theta}(x \mid a)$ for an arbitrary skew diagram $\theta$, it enables our main result in this section, namely Theorem 8.7 to be stated in simple terms. However, one can define a double Grothendieck polynomial $G_{\theta}(x ; y)$ for a skew partition $\theta$ in a similar vein, as appears in [2]. A natural conjecture would be that a result similar to Theorem 8.7 should exist relating $G_{\theta}(x ; y)$ and $G_{\theta}(x \mid y)$ for a skew partition $\theta$.

First, we need a preliminary definition before we can define the double Grothendieck polynomial $G_{\lambda}(x ; y)$ for a partition $\lambda$. So suppose $\lambda=\left(\lambda_{1}, \lambda_{2}, \ldots \lambda_{p}\right)$ is a partition. Here, we do not necessarily have $p=\ell(\lambda)$, but certainly we must have $p \geq \ell(\lambda)$. Define the permutation $w(\lambda) \in S_{\infty}=\underline{\lim } S_{n}$ by $w(\lambda)(i)=i+\lambda_{p+1-i}$ for $1 \leq i \leq p$ and $w(\lambda)(i)=i-\lambda_{i-p}^{\prime}$ for $i>p$. An explicit representation of this permutation as a product of simple reflections is constructed in Lemma 8.2, so $w(\lambda)$ is indeed a permutation. Now we can make our definition:

Definition 8.1. Define the double Grothendieck polynomial $G_{\lambda}(x ; y):=G_{w(\lambda)}(x ; y)$. Note that this definition is independent of $p$, since $G_{w}(x ; y)=G_{1^{m} \times w}(x ; y)$.

We now proceed in a similar vein to Buch [2].

Place a diagonal numbering in the boxes of $\lambda$ as follows: Number the NW-SE (defining compass directions north, west, south and east on $\lambda$ in the usual manner so that north is at the top and west is on the left) diagonals of $\lambda$ with positive integers, consecutively increasing from SW to NE, such that $p$ is the number of the main diagonal. For example with $\lambda=(4,3,1)$ and $p=4$ the numbering is explicitly shown in the following picture:

\begin{tabular}{|l|l|l|l|}
\hline 4 & 5 & 6 & 7 \\
\hline 3 & 4 & 5 & \multicolumn{1}{|c}{} \\
\cline { 1 - 2 } 2 & \multicolumn{3}{|c}{} \\
\cline { 1 - 2 } & &
\end{tabular}

Say that a partition $\mu$ contains an outer corner in the $i$-th diagonal if this diagonal contains a box outside $\mu$ such that the two boxes immediately above and to the left of it are in $\mu$. Say that $\mu$ contains an inner corner in the $i$-th diagonal if this diagonal contains a box inside $\mu$ such that the two boxes immediately below and to the right of it are not in $\mu$. So continuing with the example above, $\lambda$ contains an outer corner (among others) in the third diagonal and an inner corner in the fifth diagonal.

Suppose that $n$ is such that $n \geq p+\lambda_{1}-1$ and let $V=\bigoplus_{\mu} R\left[x_{1}, \ldots, x_{n}\right] \cdot[\mu]$ be the free $R\left[x_{1}, \ldots, x_{n}\right]$-module with basis indexed by partitions $\mu$. As in [2], we define an action of $H_{n}$ on $V$ as follows:

If $\mu$ has an outer corner in the $i$-th diagonal, set $u_{i}[\mu]=[\tilde{\mu}]$ where $\tilde{\mu}$ is the partition obtained from $\mu$ by adding a box in this corner. If $\mu$ has an inner corner in the $i$-th diagonal, set $u_{i}[\mu]=\beta[\mu]$. In all other cases, set $u_{i}[\mu]=0$. 
Lemma 8.2. We have the following representation of $w(\lambda)$ as a product of simple reflections. Let $\left(i_{1}, i_{2}, \ldots, i_{|\lambda|}\right)$ be the diagonal numbers of the boxes of $\lambda$, read one row at a time from bottom to top, reading from right to left in each row. Then $w(\lambda)=s_{i_{1}} s_{i_{2}} \ldots s_{i_{|\lambda|} \mid}$. Furthermore, this representation is minimal, that is $\ell(w(\lambda))=|\lambda|$, we have the identity $u_{w(\lambda)}[\phi]=[\lambda]$ and in any expression of the form $\beta^{t-|\lambda|} u_{w(\lambda)}=u_{i_{1}} u_{i_{2}} \ldots u_{i_{t}}$, we have $i_{t}=p$.

Proof. Suppose that $i \leq p$. Then in calculating $s_{i_{1}} s_{i_{2}} \ldots s_{i_{|\lambda|}}(i)$, the relevant simple reflections are exactly those in the $(p+1-i)$-th row of $\lambda$, so $s_{i_{1}} s_{i_{2}} \ldots s_{i_{|\lambda|}}(i)=i+\lambda_{p+1-i}$. If $i>p$, then the relevant simple reflections are exactly those in the $(i-p)$-th column of $\lambda$, so $s_{i_{1}} s_{i_{2}} \ldots s_{i_{|\lambda|}}(i)=i-\lambda_{i-p}^{\prime}$ in this case. Hence $w(\lambda)=s_{i_{1}} s_{i_{2}} \ldots s_{i_{|\lambda|}}$.

Note that $w(\lambda)(i+1)>w(\lambda)(i)$ for all $i \neq p$. Hence if $i<j$ is such that $w(\lambda)(i)>$ $w(\lambda)(j)$, we must have $i \leq p$ and $j>p$. For $i$ and $j$ in this range, $w(\lambda)(i)>w(\lambda)(j)$ if and only if $1+\lambda_{p+1-i}+\lambda_{j-p}^{\prime}>(p+1-i)+(j-p)$, which occurs if and only if $(p+1-i, j-p) \in \lambda$. Hence there are $|\lambda|$ such pairs $(i, j)$, so $\ell(w(\lambda))=|\lambda|$.

For the remaining statement, we first notice that since $w(\lambda)=s_{i_{1}} \ldots s_{i_{|\lambda|}}$, we easily calculate $u_{w(\lambda)}[\phi]=[\lambda]$. Now if $\beta^{t-|\lambda|} u_{w(\lambda)}=u_{i_{1}} u_{i_{2}} \ldots u_{i_{t}}$, then $u_{i_{1}} u_{i_{2}} \ldots u_{i_{t}}[\phi]=$ $\beta^{t-|\lambda|} u_{w(\lambda)}[\phi]=\beta^{t-|\lambda|}[\lambda] \neq 0$. Hence $u_{i_{t}}[\phi] \neq 0$, so $i_{t}=p$.

Lemma 8.3. [2] If $w \in S_{n+1}$ is such that $u_{w}[\phi] \neq 0$, then $u_{w}[\phi]=[\mu]$ for some partition $\mu$ and furthermore $w=w(\mu)$.

Proof. The proof of this lemma by induction on $\ell(w)$, and contained in [2].

We have the following immediate corollary:

Corollary 8.4. For all $h \in H_{n}$, the coefficient of $u_{w(\lambda)}$ in $h$ is equal to the coefficient of $[\lambda]$ in $h[\phi]$.

Define the products $P$ and $Q$ by

$$
\begin{aligned}
& P=\prod_{m=\infty}^{1} \prod_{i=n}^{1} h_{i}\left(x_{m} \oplus y_{m+i-p}\right) . \\
& Q=\prod_{m=k}^{1} \prod_{i=n}^{1} h_{i}\left(x_{m} \oplus y_{m+i-p}\right) .
\end{aligned}
$$

Theorem 8.5. The coefficient of $u_{w(\lambda)}$ in $P$ is the double Grothendieck polynomial $G_{\lambda}(x ; y)$. Proof. This follows from Lemma 7.11 and Lemma 8.2, noting that factors on the left hand side of (7.13) with $m \leq 0$ are either one, or do not contain $u_{p}$.

Theorem 8.6. The coefficient of $[\lambda]$ in $Q[\phi]$ is the factorial Grothendieck polynomial $G_{\lambda}(x \mid y)$ in $x_{1}, \ldots x_{k}$. 
Proof. Expand $Q$, and note that each term is a product of terms of the form $\left(x_{m} \oplus\right.$ $\left.y_{m+i-p}\right) u_{i}$. Write this product as

$$
\prod_{j=1}^{q}\left(x_{m_{j}} \oplus y_{m_{j}+i_{j}-p}\right) u_{i_{j}}
$$

If $\left(\prod_{j=1}^{q} u_{i_{j}}\right)[\phi] \neq 0$, then we can interpret this product in the following way:

Form the tableau $T$ by placing $m_{j}$ in the inner corner in diagonal number $i_{j}$ of the partition $u_{i_{j}} \ldots u_{i_{q}}[\phi]$ for $j=1,2, \ldots q$. These numbers are added in nondecreasing order, and the occurrences of each number $i$ are added from SW to NE. Furthermore, at all stages during the addition process, the shape of all the numbers added up to that point is a partition. So $T$ is a semistandard set-valued tableau with entries from $[n]$. Note that if $\alpha$ is a cell with diagonal number $i$, then $c(\alpha)=i-p$. Hence we can write

$$
\left(\prod_{j=1}^{q} u_{i_{j}}\right)[\phi]=\beta^{|T|-|\lambda|}\left(\prod_{\substack{\alpha \in \lambda \\ r \in T(\alpha)}} x_{r} \oplus y_{r+c(\alpha)}\right) u_{w(\lambda)}
$$

for some partition $\lambda$ for which $T$ is a $\lambda$-tableau.

Upon considering all such terms in $Q$, it becomes evident that the coefficient of $[\lambda]$ in $Q[\phi]$ is the factorial Grothendieck polynomial $G_{\lambda}(x \mid y)$ as required.

Note that $\lim _{k \rightarrow \infty} Q=P$. Hence, the preceding three results give us the following theorem.

Theorem 8.7 (Relationship between factorial and double Grothendieck polynomials).

$$
G_{\lambda}(x ; y)=\lim _{k \rightarrow \infty} G_{\lambda}\left(x_{1}, \ldots, x_{k} \mid y\right)
$$

\section{Acknowledgements}

The author would like to thank A. Molev for his guidance through this area of study, and also A. Henderson, for his help with preparation of the manuscript.

\section{References}

[1] Bourbaki, N, “Groupes et algèbres de Lie," Ch 4-6, Hermann, Paris 1968.

[2] A. S. Buch, A Littlewood-Richardson Rule for the K-Theory of Grassmannians, Acta. Math, 189 (2002), 37-78.

[3] S. Fomin and C. Greene. Noncommutative Schur functions and their applications, Discrete Math, 193, (1998), 179-200. 
[4] S. Fomin and A. N. Kirillov, Grothendieck polynomials and the Yang-Baxter equation, Proc. Formal Power Series and Alg. Comb, (1994), 183-190.

[5] S. Fomin and A. N. Kirillov, The Yang-Baxter equation, symmetric functions and Schubert polynomials, Discrete Math, 153 (1996) 123-143.

[6] V. Gasharov, A short proof of the Littlewood-Richardson rule, European J. Combin. 19 (1998) 451-453.

[7] G. D. James and M. H. Peel, Specht series for skew representations of symmetric groups, J. Algebra 56 (1979), 343-364.

[8] D. E. Littlewood and A. R. Richardson, Group characters and algebra, Philos. Trans. Roy. Soc. London Ser. A 233 (1934), 49-141.

[9] A. Lascoux, "Interpolation", Lectures at Tianjin University, June 1996.

[10] A. Lascoux and M. P. Schützenberger, Structure de Hopf de l'anneau de cohomologie et de l'anneau de Grothendieck d'une variété de drapeaux, C.R. Acad. Sci. Parix Sér. I Math, 295 (1982), 629-633.

[11] C. Lenart, Combinatorial Aspects of the K-Theory of Grassmannians, Ann. Comb, 4 (2000), 67-82.

[12] I. G. Macdonald, Schur functions: theme and variations, in "Actes 28-e Séminaire Lotharingien", Publ. I.R.M.A. Strasbourg, 1992, 498/S-27, 5-39.

[13] I. G. Macdonald, "Symmetric Functions and Hall Polynomials," 2nd edition, Oxford University Press, Oxford 1995.

[14] A. I. Molev and B. E. Sagan, A Littlewood-Richardson Rule For Factorial Schur Functions, Trans. Amer. Math. Soc, 351 (1999), 4429-4443.

[15] A. Molev, Factorial Supersymmetric Schur Functions and Super Capelli Identities, Amer. Math. Soc. Transl, 181 (1998), 109-137.

[16] A. Okounkov, Quantum immanants and higher Capelli identities, Transformation Groups 1 (1996) 99-126.

[17] B. E. Sagan, "The symmetric group: representations, combinatorial algorithms, and symmetric functions," 2nd edition, Springer, New York 2001.

[18] A. V. Zelevinsky, A generalization of the Littlewood-Richardson rule and the Robinson-Schensted-Knuth correspondence, J. Algebra 69 (1981), 82-94. 